\title{
On the conjectures of Gross and Leopoldt
}

\author{
Soogil SeO
}

\begin{abstract}
We show that the Leopoldt conjecture implies the so-called Hilbert's theorem 90 for compact modules made from the $p$-units over the cyclotomic $\mathbb{Z}_{p}$-extension $k_{\infty}^{\text {cyc }}$ of $k$. And under the generalized Gross conjecture, we show that Hilbert's theorem 90 for the compact modules above is equivalent to the affirmation of the Leopoldt conjecture.
\end{abstract}

\section{Introduction}

Let $k$ be a number field and let $p$ be a prime number. The purpose of this paper is to show that two basic conjectures of Gross and Leopoldt are related through arithmetic properties of certain compact modules made from the $p$-units which is the so-called Hilbert's theorem 90 over the cyclotomic $\mathbb{Z}_{p^{-}}$ extension $k_{\infty}^{\mathrm{cyc}}$ of $k$.

More precisely we prove that under the generalized Gross conjecture, the Leopoldt conjecture is equivalent to the so-called Hilbert's theorem 90 for a closure of the $p$-units over $k_{\infty}^{\text {cyc }} / k$. The arithmetic property which is called the universal norm property of the topological closure of the $p$-units over $k_{\infty}^{\text {cyc }} / k$ will play a role in connecting two conjectures via class field theory.

We will explain very briefly the general strategy to relate the two conjectures. The Leopoldt conjecture concerns on the ranks of two modules $\mathcal{U}_{n}(p)$ and $\overline{\Delta\left(U_{n}(p)\right)}$ made from the $p$-units $U_{n}(p)$ of $k_{n}$ where $k_{n}$ is the unique subfield of $k_{\infty}^{\text {cyc }}$ of degree $p^{n}$ over $k$. Through the weak Leopoldt conjecture and Hilbert's theorem 90 over $k_{\infty}^{\text {cyc }} / k$, the Leopoldt conjecture reduces to the ranks of the universal norms $\mathcal{U}_{n}(p)^{\text {univ }}$ and ${\overline{\Delta\left(U_{n}(p)\right)}}^{\text {univ }}$. These universal norm groups finally can be related to the Tate module $T_{p}(k)$ which is related to the generalized Gross conjecture through infinite class field theory.

In order to state the main theorem, we recall some definitions briefly. For the cyclotomic $\mathbb{Z}_{p}$-extension $k_{\infty}^{\text {cyc }}$, write $k_{\infty}^{\text {cyc }}=\bigcup_{n \geq 0} k_{n}$ with $k_{n}$ the unique subfield of $k_{\infty}^{\text {cyc }}$ of degree $p^{n}$ over $k$. Let $\Gamma$ denote the procyclic group

This work was supported by the National Research Foundation of Korea(NRF) grant funded by the Korea government(MSIP)(No. 2014001824). 
$G\left(k_{\infty}^{\text {cyc }} / k\right)$ generated by $\gamma$ and for each $n \geq 0$, let $\Gamma_{n}=G\left(k_{\infty}^{\text {cyc }} / k_{n}\right)$ be the unique subgroup of $\Gamma$ with index $p^{n}$. We define the Tate module $T_{p}(k)$ of $k$ to be

$$
T_{p}(k)={\underset{n}{n}}_{\lim } G\left(K_{n} / k_{n}\right)
$$

the inverse limit of $G\left(K_{n} / k_{n}\right)$ where $K_{n}$ is the maximal abelian unramified $p$-extension of $k_{n}$ such that all primes dividing $p$ split completely. One of the equivalent forms of the generalized Gross conjecture can be described via class field theory as the finiteness of the Galois coinvariant

$$
T_{p}(k)_{\Gamma}:=T_{p}(k) /(\gamma-1) T_{p}(k)
$$

of the Tate module $T_{p}(k)$ over $k_{\infty}^{\text {cyc }} / k$ (cf. Theorem 1.14 of [17]). For a finite set $A$, let $\sharp(A)$ denote the cardinality of $A$.

The generalized Gross conjecture for $(k, p)$ : Let $k$ be a number field. Then

$$
\sharp\left(T_{p}(k)_{\Gamma}\right)<\infty .
$$

We will state some equivalent forms of the Leopoldt conjecture. Let $\delta$ denote the diagonal embedding

$$
\delta: k^{\times} \longrightarrow \prod_{v \mid p} k_{v}^{\times}, \alpha \mapsto(\alpha, \ldots, \alpha) .
$$

Let $U_{k}(p)$ be the $p$-units of $k$ and let $\overline{\delta\left(U_{k}(p)\right)}$ be the topological $p$ adic closure of $\delta\left(U_{k}(p)\right)$ in $\prod_{v \mid p} k_{v}^{\times}$. For each prime $v$ of $k$ dividing $p$, let $U_{v}^{1}=1+\mathfrak{p}_{v}$ denote the principal units at $v$.

If we denote by $r_{1}$ and $r_{2}$ respectively the number of real and complex places of $k$, then the Leopoldt conjecture is equivalent to the $\mathbb{Z}_{p}$-rank $\operatorname{rank}_{\mathbb{Z}_{p}}\left(\overline{\delta\left(U_{k}(p)\right)} \cap \prod_{v \mid p} U_{v}^{1}\right)$ of the $p$-part of $\overline{\delta\left(U_{k}(p)\right)}$ is $r_{1}+r_{2}-1$ (see Conjecture 7.9 of p. 102 of [21])

$$
\operatorname{rank}_{\mathbb{Z}_{p}}\left(\overline{\delta\left(U_{k}(p)\right)} \cap \prod_{v \mid p} U_{v}^{1}\right)=r_{1}+r_{2}-1 .
$$

For a $\mathbb{Z}$-module $M$, let

$$
\widehat{M}={\underset{\varliminf}{\leftarrow}}_{\lim _{n}} M / M^{p^{n}}
$$


denote the $p$-adic closure of $M$. Let $\Delta$ be the induced map

$$
\Delta: k^{\times} \longrightarrow \prod_{v \mid p} \widehat{k}_{v}^{\times}, \alpha \mapsto(\alpha, \ldots, \alpha) .
$$

Then the topological closure $\overline{\Delta\left(U_{k}(p)\right)}$ of the image $\Delta\left(U_{k}(p)\right)$ of $U_{k}(p)$ under $\Delta$ in the group $\prod_{v \mid p} \widehat{k}_{v}^{\times}$is equal to the image of the induced map

$$
\widehat{\Delta}: \mathcal{U}_{k}(p) \longrightarrow \prod_{v \mid p} \widehat{k}_{v}^{\times}
$$

where $\mathcal{U}_{k}(p):=U_{k}(p) \otimes_{\mathbb{Z}} \mathbb{Z}_{p}$.

The Leopoldt conjecture for $(k, p)$ : Let $k$ be a number field. Then $\widehat{\Delta}$ induces the following isomorphism

$$
\mathcal{U}_{k}(p) \cong \overline{\Delta\left(U_{k}(p)\right)}
$$

Equivalently, the Leopoldt conjecture can be stated in terms of the $\mathbb{Z}_{p^{-}}$ $\operatorname{rank}$ of $\overline{\Delta\left(U_{k}(p)\right)}$

$$
\operatorname{rank}_{\mathbb{Z}_{p}}\left(\overline{\Delta\left(U_{k}(p)\right)}\right)=r_{1}+r_{2}+r-1
$$

where $r=r_{k}(p)$ denotes the number of primes of $k$ dividing $p$ (cf. Theorem 10.3.6 and Remark of page 634 of [22]). Note that the $p$-adic closure of $\overline{\delta\left(U_{k}(p)\right)}$ in $\prod_{v \mid p} \widehat{k}_{v}^{\times}$is equal to $\overline{\Delta\left(U_{k}(p)\right)}$.

We will define Hilbert's theorem 90 for compact modules over a $\mathbb{Z}_{p^{-}}$ extension $k_{\infty}$ of $k$.

Over a $\mathbb{Z}_{p^{-}}$-extension $k_{\infty}=\bigcup_{n>0} k_{n}$ of $k$, let $N_{m, n}=N_{k_{m} / k_{n}}$ denote the norm map from $k_{m}$ to $k_{n}$ and let $\bar{N}_{m}=N_{m, 0}$ denote the norm map from $k_{m}$ to the ground field $k_{0}=k$.

For any multiplicative subgroup $M_{n}$ of the intermediate field $k_{n}^{\times}$, we define the norm compatible subgroups $M_{n}^{\text {comp }}$ and the universal norm subgroups $M_{n}^{\text {univ }}$ as follows

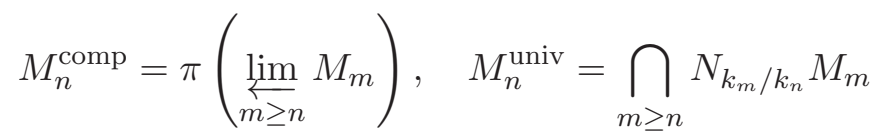

where the inverse limits are taken with respect to the norm maps and $\pi=\pi_{n}$ denotes the natural projection from $\lim _{m \geq n} M_{m}$ to $M_{n}$ defined as $\pi\left(\left(b_{m}\right)_{m \geq n}\right)=b_{n}$. We will define similar notions by tensoring with $\mathbb{Z}_{p}$. 
Let $A_{n}$ be a compact $\mathbb{Z}_{p}\left[G\left(k_{n} / k\right)\right]$-module with the norm maps for all $m \geq n$,

$$
N_{m, n}: A_{m} \longrightarrow A_{n}
$$

If we define $A_{\infty}:=\lim _{n \geq 0} A_{n}$ to be the inverse limits of $\left\{A_{n}\right\}_{n \geq 0}$ with respect to the norm maps $N_{m, n}$, then $A_{\infty}$ is a $\Lambda:=\lim _{n \geq 0} \mathbb{Z}_{p}\left[G\left(k_{n} / k\right)\right]$ module. Let

$$
\left(A_{\infty}\right)_{\Gamma}=A_{\infty} /(\gamma-1) A_{\infty}
$$

Definition 1.1. Let $k_{\infty}=\bigcup_{n \geq 0} k_{n}$ be a $\mathbb{Z}_{p}$-extension of $k$.

(i) We will say that $A_{k}$ satisfy Hilbert's theorem 90 over $k_{\infty} / k$,

$$
\left(A_{\infty}\right)_{\Gamma} \cong A_{k}^{\text {univ }}
$$

(ii) We will say the generalized Gross conjecture holds for $\left(k_{\infty}, p\right)$ if the generalized Gross conjecture holds for $\left(k_{n}, p\right)$ for all $n \geq 0$.

(iii) We will say the Leopoldt conjecture holds for $\left(k_{\infty}, p\right)$ if the Leopoldt conjecture holds for $\left(k_{n}, p\right)$ for all $n \geq 0$.

For a Galois extension $k^{\prime} / k$ and their global $p$-units $U_{k^{\prime}}(p)$ and $U_{k}(p)$ of $k^{\prime}$ and $k$ respectively, we have the induced norm map $N_{k^{\prime} / k}: U_{p}\left(k^{\prime}\right) \otimes_{\mathbb{Z}} \mathbb{Z}_{p} \longrightarrow$ $U_{k}(p) \otimes_{\mathbb{Z}} \mathbb{Z}_{p}$ such that $N_{k^{\prime} / k}$ acts trivially on $\mathbb{Z}_{p}$, i.e., for $a \in k^{\prime}$ and $\alpha \in \mathbb{Z}_{p}$,

$$
N_{k^{\prime} / k}(a \otimes \alpha)=N_{k^{\prime} / k}(a) \otimes \alpha .
$$

For the global $p$-units $U_{n}(p)=U_{k_{n}}(p)$ of $k_{n}$, write

$$
\mathcal{U}_{n}(p):=U_{n}(p) \otimes_{\mathbb{Z}} \mathbb{Z}_{p}, \quad \mathcal{U}_{\infty}:=\lim _{n \geq 0}\left(U_{n}(p) \otimes_{\mathbb{Z}} \mathbb{Z}_{p}\right)
$$

with $U_{0}(p)=U_{k}(p), \mathcal{U}_{0}(p)=\mathcal{U}_{k}(p)$. The group $\mathcal{U}_{k}(p)^{\text {univ }}$ of universal norm elements is defined as

$$
\mathcal{U}_{k}(p)^{\text {univ }}=\bigcap_{n \geq 0} N_{n} \mathcal{U}_{n}(p)=\bigcap_{n \geq 0} N_{n}\left(U_{n}(p) \otimes_{\mathbb{Z}} \mathbb{Z}_{p}\right)=\bigcap_{n \geq 0}\left(N_{n}\left(U_{n}(p)\right) \otimes_{\mathbb{Z}} \mathbb{Z}_{p}\right)
$$

The projection $\pi$ induces the following natural map

$$
\pi:\left(\mathcal{U}_{\infty}\right)_{\Gamma} \rightarrow \mathcal{U}_{k}(p)
$$

such that the image of $\pi$ is equal to the norm comparable elements $\mathcal{U}_{k}(p)^{\text {comp }}$ of $\mathcal{U}_{k}(p)$ where $\left(\mathcal{U}_{\infty}\right)_{\Gamma}$ is the coinvariant of $\mathcal{U}_{\infty}$ by $\Gamma=G\left(k_{\infty} / k\right)$. Then by 
an argument of compactness, it follows that $\mathcal{U}_{k}(p)^{\text {comp }}=\mathcal{U}_{k}(p)^{\text {univ }}$. We state the main theorem of this paper.

Theorem 1.2. (i) The Leopoldt conjecture for $(k, p)$ implies Hilbert's theorem 90 for $\overline{\Delta\left(U_{k}(p)\right)}$ over $k_{\infty}^{\mathrm{cyc}} / k$.

(ii) Under the generalized Gross conjecture for $\left(k_{\infty}^{\mathrm{cyc}}, p\right)$, Hilbert's theorem 90 for $\overline{\Delta\left(U_{n}(p)\right)}$ over $k_{\infty}^{\mathrm{cyc}} / k_{n}$ for all $n \geq 0$ is equivalent to the affirmation of the Leopoldt conjecture for $\left(k_{\infty}^{\mathrm{cyc}}, p\right)$.

When $k$ is totally real or a CM-field, we have the following corollary.

Corollary 1.3. (i) If $k$ is a totally real field then the affirmation of the Leopoldt conjecture for $\left(k_{\infty}^{\mathrm{cyc}}, p\right)$ is equivalent to the affirmation of the Gross conjecture for $\left(k_{\infty}^{\text {cyc }}, p\right)$ and Hilbert's theorem 90 for $\overline{\Delta\left(U_{n}(p)\right)}$ over $k_{\infty}^{\mathrm{cyc}} / k_{n}$ for all $n \geq 0$.

(ii) If $k$ is a CM-field with a maximal real subfield $k^{+}$then the affirmation of the Leopoldt conjecture for $\left(k_{\infty}^{\mathrm{cyc}}, p\right)$ is equivalent to the affirmation of the Gross conjecture for $\left(k_{\infty}^{+\mathrm{cyc}}, p\right)$ and Hilbert's theorem 90 for $\overline{\Delta\left(U_{k_{n}^{+}}(p)\right)}$ over $k_{\infty}^{+ \text {cyc }} / k_{n}^{+}$for all $n \geq 0$.

As special cases of Corollary 1.3, if the number $r_{\infty}(p)$ (resp. $r_{\infty^{+}}(p)$ ) of primes of $k_{\infty}^{\text {cyc }}$ (resp. $k_{\infty}^{+ \text {cyc }}$ ) dividing $p$ is one, then we have more simple descriptions.

(i) If $k$ is a totally real field with $r_{\infty}(p)=1$, then the affirmation of the Leopoldt conjecture for $\left(k_{\infty}^{\text {cyc }}, p\right)$ is equivalent to Hilbert's theorem 90 for $\overline{\Delta\left(U_{n}(p)\right)}$ over $k_{\infty}^{\text {cyc }} / k_{n}$ for all $n \geq 0$.

(ii) If $k$ is a CM-field with the maximal real subfield $k^{+}$such that $r_{\infty^{+}}(p)=$ 1 , then the affirmation of the Leopoldt conjecture for $\left(k_{\infty}^{\mathrm{cyc}}, p\right)$ is equivalent to Hilbert's theorem 90 for $\overline{\Delta\left(U_{k_{n}^{+}}(p)\right)}$ over $k_{\infty}^{+ \text {cyc }} / k_{n}^{+}$for all $n \geq 0$.

In $\S 2$, we recall the generalized Gross conjecture and the Leopoldt conjecture. In $\S 3$, we prove Theorem 1.2 and Corollary 1.3.

\section{Conjectures of Gross and Leopoldt}

Leopoldt made his conjecture for the units of a totally real field, and Gross made his conjecture for the minus part of the $p$-units of a CM field. Two conjectures were generalized independently to a number field $k$. Let $k_{\infty}=$ 
$\bigcup_{n \geq 0} k_{n}$ be a $\mathbb{Z}_{p^{-}}$extension of $k$ with $k_{n}$ the unique subfield of $k_{\infty}$ of degree $p^{n}$ over $k$ and $G_{n}=G\left(k_{n} / k\right)$.

Following Iwasawa (see $\S 4$ of [13]), we briefly explain how the two conjectures are connected using the cohomologies over $\Gamma=G\left(k_{\infty} / k\right)$. From the seven term exact sequence of Auslander-Brumer and Chase-HarrisonRosenberg, one can define

$$
\psi_{S}: H^{2}\left(\Gamma, U_{\infty}(S)\right) \longrightarrow B_{S}:=\operatorname{ker}\left(H^{2}\left(\Gamma, k_{\infty}^{\times}\right) \rightarrow H^{2}\left(\Gamma, I_{\infty}\right)\right)
$$

where $U_{\infty}(S)$ and $I_{\infty}$ are the group of the $S:=\{v \mid p\}$-units and the group of fractional ideals of $k_{\infty}$ respectively (see Proposition 2.1 of [5] or Proposition 1 of [13]). Then there are three equivalent conditions;

i) $\psi_{S}$ is surjective,

ii) $C l_{\infty}(S)^{\Gamma}<\infty$,

iii) $H^{1}\left(\Gamma, C l_{\infty}(S)\right)=0$,

where $C l_{\infty}(S)$ is the $p$-part of the $S$-ideal class group of $k_{\infty}$

If $k_{\infty}$ is equal to the cyclotomic $\mathbb{Z}_{p}$-extension $k_{\infty}^{\text {cyc }}$, then the generalized Gross conjecture for $(k, p)$ is equivalent to one of the three conditions (i), (ii) and (iii) above. When $k$ is a totally real field, the Leopoldt conjecture, i.e., the uniqueness of a $\mathbb{Z}_{p}$-extension of $k$, implies $\psi_{S}$ is surjective. When $k$ is a CM field and $p$ is odd, there exists a decomposition of \pm 1 -eigenspaces via the complex conjugation,

$$
\psi_{S}^{ \pm}: H^{2}\left(\Gamma, U_{\infty}(S)\right)^{ \pm} \longrightarrow B_{S}^{ \pm}
$$

The Leopoldt conjecture implies the surjectivity of the plus part $\psi_{S}^{+}$as above and the surjectivity of the minus part $\psi_{S}^{-}$is the Gross conjecture. Hence when $k$ is a CM field, the conjectures of Leopoldt and Gross implies the generalized Gross conjecture.

\section{1 .}

We introduce briefly the generalized Gross conjecture with its reformulation in terms of the Tate module. For an arbitrary number field $k$, let $S$ denote the set of the primes of $k$ dividing $p$. We denote by $U_{k}(p)$ the group of $S$-units of $k$.

For each finite prime $v$ of $k$, let $k_{v}^{\text {univ }}$ denote the group of universal norms from the cyclotomic $\mathbb{Z}_{p}$-extension $k_{v, \infty}^{\text {cyc }}$ of the local field $k_{v}$. Let $\log _{p}$ denote 
Iwasawa's p-adic logarithm normalized by $\log _{p}(p)=0$. Thus it induces an isomorphism from $\mathbb{Q}_{p}^{\times} / p^{\mathbb{Z}} \mu\left(\mathbb{Q}_{p}\right)=1+p \mathbb{Z}_{p}$ to $p \mathbb{Z}_{p}$ where $\mu\left(\mathbb{Q}_{p}\right)$ denotes the torsion subgroup of $\mathbb{Q}_{p}^{\times}$. It also follows that for each $v \mid p$,

$$
a \in k_{v}^{\text {univ }} \Leftrightarrow a \in \operatorname{ker}\left(\log _{p} \circ N_{k_{v} / \mathbb{Q}_{p}}\right) .
$$

Let

$$
\lambda_{k}=\sum_{v \mid p} \log _{p} \circ N_{k_{v} / \mathbb{Q}_{p}}: k^{\times} \rightarrow \oplus_{v \mid p} \log _{p} N_{k_{v} / \mathbb{Q}_{p}}\left(k_{v}^{\times}\right) \cdot v \cong \prod_{v \mid p} k_{v}^{\times} / k_{v}^{\text {univ }}
$$

be the map defined by $\lambda_{k}(a)=\sum_{v \mid p} \log _{p} \circ N_{k_{v} / \mathbb{Q}_{p}}(a) v$.

If we restrict $\lambda_{k}$ to $U_{k}(p)$ and extend it $U_{k}(p) \otimes \mathbb{Z}_{p}$ by $\mathbb{Z}_{p}$-linearity, then we can obtain a homomorphism $g_{k}$ of $\mathbb{Z}_{p^{-} \text {-modules }}$

$$
g_{k}: U_{k}(p) \otimes \mathbb{Z}_{p} \longrightarrow \oplus_{v \mid p} \log _{p} \circ N_{k_{v} / \mathbb{Q}_{p}}\left(k_{v}^{\times}\right) \cdot v .
$$

Let $r=r_{k}(p)$ denote the number of primes of $k$ dividing $p$. By the product formula of the Artin map, the image of $g_{k}$ has a $\mathbb{Z}_{p}$-rank at most $r-1$ (see page 455 of [6] and page 11 of [17]). Let $d_{k}(p) \geq 0$ be the nonnegative integer such that

$$
d_{k}(p)=r-1-\operatorname{rank}\left(\operatorname{im}\left(g_{k}\right)\right) .
$$

Let $r_{1}$ and $r_{2}$ denote respectively the number of real and complex primes of $k$. Notice that by Dirichlet's unit theorem, $\mathbb{Z}_{p}$-rank rank $\left(\operatorname{ker}\left(g_{k}\right)\right)$ of the kernel of $g_{k}$ is given by

$$
\operatorname{rank}\left(\operatorname{ker}\left(g_{k}\right)\right)=r_{1}+r_{2}+d_{k} .
$$

The generalized Gross conjecture for $(k, p)$ is then equivalent to

$$
d_{k}(p)=0 .
$$

This is an extension of a conjecture of Gross (see [10]) due to Jaulent (see [14] and [17]). In fact, Theorem 1.14 of [17] shows that the condition $d_{k}(p)=0$ is equivalent to one of the three conditions (i), (ii) and (iii) defined in the beginning of $\S 2$ for $k_{\infty}=k_{\infty}^{\text {cyc }}$. For absolute abelian fields, the proof of Gross conjecture is due to Greenberg (see [8]).

Let $k_{\infty}=k_{\infty}^{\text {cyc }}=\bigcup_{n \geq 0} k_{n}$ the cyclotomic $\mathbb{Z}_{p}$-extension of a number field $k=k_{0}$ with $k_{n}$ the unique subfield of $k_{\infty}$ of degree $p^{n}$ over $k$. Let $\Gamma$ denote 
the procyclic group $G\left(k_{\infty} / k\right)$ and for each $n \geq 0$, let $\Gamma_{n}=G\left(k_{\infty} / k_{n}\right)$ be the unique subgroup of $\Gamma$ with index $p^{n}$. We recall the Tate module

$$
T_{p}(k)=\underbrace{\lim }_{n} G\left(K_{n} / k_{n}\right)
$$

of $k$ where $K_{n}$ is the maximal abelian unramified $p$-extension of $k_{n}$ such that all primes dividing $p$ split completely.

We now introduce the following equivalence of the generalized Gross conjecture for $(k, p)$

$$
\sharp\left(T_{p}(k)_{\Gamma}\right)<\infty .
$$

This follows from the following well known proposition using class field theory (see Proposition of 1.2 of [17]).

$$
d_{k}(p)=0 \text { if and only if } \sharp\left(T_{p}(k)_{\Gamma}\right)<\infty .
$$

The generalized Gross conjecture can also be described in terms of the invariant of the Tate module, i.e.,

$$
\sharp\left(T_{p}(k)^{\Gamma}\right)<\infty
$$

which follows from the identity $\operatorname{rank} T_{p}(k)_{\Gamma}=\operatorname{rank} T_{p}(k)^{\Gamma}$. This follows directly from the finiteness rank $T_{p}(k)<\infty$ (see Theorem 5 of $\S 3$ of [12]) and the exact sequence

$$
1 \longrightarrow T_{p}(k)^{\Gamma} \longrightarrow T_{p}(k) \stackrel{\gamma-1}{\longrightarrow} T_{p}(k) \longrightarrow T_{p}(k)_{\Gamma} \longrightarrow 1 .
$$

\section{Proof of Theorem 1.2}

As explained in the introduction, the general strategy to relate the two conjectures is applying infinite class field theory over $k_{\infty}^{\text {cyc }}=\bigcup_{n \geq 0} k_{n}$. The Leopoldt conjecture which concerns on the ranks of two modules $\mathcal{U}_{n}(p)$ and $\overline{\Delta\left(U_{n}(p)\right)}$ reduces to the ranks of the universal norms $\mathcal{U}_{n}(p)^{\text {univ }}$ and ${\overline{\Delta\left(U_{n}(p)\right)}}^{\text {univ }}$ through the weak Leopoldt conjecture and Hilbert's theorem 90 over $k_{\infty}^{\text {cyc }} / k$. These universal norm groups finally are related to $T_{p}(k)$ which can be related to the generalized Gross conjecture through infinite class field theory.

In $\S \S 3.1$ and 3.2 , we recall the condition so-called (HNT) for $p$-units and respectively Hilbert's theorem 90 over a procyclic extension.

We prove Theorem 1.2 in the separated subsections. In $\S 3.3$, we prove that the affirmation of the generalized Gross conjecture for $\left(k_{\infty}^{\mathrm{cyc}}, p\right)$ and 
Hilbert's theorem 90 for $\overline{\Delta\left(U_{n}(p)\right)}$ over $k_{\infty}^{\text {cyc }} / k$ for all $n \geq 0$ implies the affirmation of the Leopoldt conjecture for $\left(k_{\infty}^{\mathrm{cyc}}, p\right)$. In $\S 3.4$, we prove the first claim of Theorem 1.2 that the affirmation of the Leopoldt conjecture for $(k, p)$ implies Hilbert's theorem 90 for $\overline{\Delta\left(U_{k}(p)\right)}$ over $k_{\infty}^{\text {cyc }} / k$. This will complete the proof of Theorem 1.2.

In $\S 3.5$, we prove Corollary 1.3 of the introduction and explain special cases of the corollary. In $\S 3.6$, we explain that results of Jaulent can also be used to prove part of Theorem 1.2.

\section{1.}

In this subsection, we will define a condition for the $p$-units which will be called simply as (HNT) over a procyclic extension.

Let $K / k$ be a finite cyclic extension and for a finite set $S$ of primes of $k$ containing all ramified primes in $K / k$, let $U_{k}(S)$ be the global $S$-units of $k$. Let

$$
S^{\prime}=\{w \mid v ; v \in S\}
$$

be the set of primes of $K$ lying over each prime $v \in S$. We also let $U_{K}(S):=$ $U_{K}\left(S^{\prime}\right)$ denote the global $S^{\prime}$-units of $K$. Let

$$
J_{K, S}:=\prod_{w \notin S^{\prime}} U_{v} \times \prod_{w \in S^{\prime}} k_{v}^{\times}
$$

be the $S$-idele group and let

$$
C_{K, S}:=J_{K, S} / U_{K}(S)
$$

be the $S$-idele class group where we identify $U_{K}(S)$ with a subgroup of $J_{K, S}$ via the the diagonal embedding

$$
\phi_{K, S}: U_{K}(S) \longrightarrow J_{K, S}
$$

Since $w \notin S$ is unramified in $K / k$, the local units $U_{w}$ is cohomologically trivial for $w \notin S$. Hence by Shapiro's lemma, we have

$$
\begin{aligned}
\widehat{H}^{i}\left(G(K / k), J_{K, S}\right) & \cong \widehat{H}^{i}\left(G(K / k), \prod_{w \in S^{\prime}} K_{w}\right) \\
& \cong \prod_{v \in S} \widehat{H}^{i}\left(G(K / k), \operatorname{Ind}_{G(K / k)}^{G\left(K_{w} / k_{v}\right)}\left(K_{w}\right)\right) \\
& \cong \prod_{v \in S} \widehat{H}^{i}\left(G\left(K_{w} / k_{v}\right), K_{w}^{\times}\right)
\end{aligned}
$$


where $w$ is a chosen prime of $K$ above $v$.

Using the isomorphism above and Hilbert's theorem 90, the exact sequence $1 \longrightarrow U_{K}(S) \stackrel{\phi_{K, S}}{\longrightarrow} J_{K, S} \longrightarrow C_{K, S} \longrightarrow 1$ leads to

$$
1 \longrightarrow \widehat{H}^{-1}\left(G_{K / k}, C_{K, S}\right) \longrightarrow \frac{U_{k}(S)}{N_{K / k} U_{K}(S)} \stackrel{\tilde{\phi}_{K / k, S}}{\longrightarrow} \prod_{v \in S} \widehat{H}^{0}\left(G_{K_{w} / k_{v}}, K_{w}^{\times}\right)
$$

where $N_{K / k}$ is the norm map of $K / k$ and $\tilde{\phi}_{K / k, S}$ is the map induced from the diagonal embedding $\phi_{K, S}$. It follows that

$$
\widehat{H}^{-1}\left(G_{K / k}, C_{K, S}\right) \cong \operatorname{ker}\left(\tilde{\phi}_{K / k, S}\right)
$$

We let

$$
\phi_{K / k, S}: U_{k}(S) \longrightarrow \prod_{w \in S} \widehat{H}^{0}\left(G_{K_{w} / k_{v}}, K_{w}^{\times}\right) \cong \prod_{w \in S} k_{v}^{\times} / N_{K_{w} / k_{v}} K_{w}^{\times}
$$

denote the composition of the natural projection $\pi: U_{k}(S) \longrightarrow U_{k}(S) /$ $N_{K / k} U_{K}(S)$ and $\tilde{\phi}_{K / k, S}: U_{k}(S) / N_{K / k} U_{K}(S) \longrightarrow \prod_{w \in S} \widehat{H}^{i}\left(G_{K_{w} / k_{v}}, K_{w}^{\times}\right)$. (HNT) for $k^{\times}$implies that

$$
N_{K / k} U_{K}(S) \subset \operatorname{ker}\left(\phi_{K / k, S}\right) \subset U_{k}(S) \cap N_{K / k} K^{\times} .
$$

Definition 3.1. For a finite cyclic extension $K / k$, if $\tilde{\phi}_{K / k, S}$ is injective, i.e.,

$$
\operatorname{ker}\left(\phi_{K / k, S}\right)=N_{K / k} U_{K}(S),
$$

then we say that $U_{k}(S)$ satisfies (HNT) over $K / k$.

We extend the definition into an infinite Galois extension such that the Galois group is an infinite procyclic group, a topological closure of an infinite cyclic group.

For an infinite procyclic extension $K / k$, let

$$
\operatorname{ker}\left(\phi_{K / k, S}\right):=\bigcap_{L} \operatorname{ker}\left(\phi_{L / k, S}\right), \quad U_{k}(S)^{\mathrm{univ}}:=\bigcap_{L} N_{L / k} U_{L}(S)
$$

for all finite cyclic subextensions $L$ of $K / k$. For a finitely generated $\mathbb{Z}$-module $M$, let $M / \operatorname{tor}(M)$ be the quotient of $M$ by its torsion $\operatorname{tor}(M)$ and let

$$
\operatorname{rank}_{\mathbb{Z}}(M):=\operatorname{rank}_{\mathbb{Z}}(M / \operatorname{tor}(M))
$$

be the $\mathbb{Z}$-rank of $M / \operatorname{tor}(M)$. 
Definition 3.2. For an infinite procyclic extension $K / k$, if

$$
\operatorname{rank}_{\mathbb{Z}}\left(\operatorname{ker}\left(\phi_{K / k, S}\right)\right)=\operatorname{rank}_{\mathbb{Z}}\left(U_{k}(S)^{\text {univ }}\right),
$$

then we say that $U_{k}(S)$ satisfies (HNT) over $K / k$.

If we denote by $\operatorname{ker}\left(\phi_{K / k, S}\right)$ the kernel of $\phi_{K / k, S}$, then we have

$$
1 \longrightarrow \operatorname{ker}\left(\phi_{K / k, S}\right) \otimes \mathbb{Z}_{p} \longrightarrow U_{k}(S) \otimes \mathbb{Z}_{p} \longrightarrow J_{K, S}^{G(K / k)} / N_{K / k} J_{K, S} \otimes \mathbb{Z}_{p}
$$

by tensoring with $\mathbb{Z}_{p}$ to the following exact sequence,

$$
1 \longrightarrow \operatorname{ker}\left(\phi_{K / k, S}\right) \longrightarrow U_{k}(S) \longrightarrow J_{K, S}^{G(K / k)} / N_{K / k} J_{K, S}
$$

If we denote by $\bar{\phi}_{K / k, S}:=\phi_{K / k, S} \otimes 1$ the map induced from $\phi_{K / k, S}$, then the exact sequence above shows that

$$
\operatorname{ker}\left(\bar{\phi}_{K / k, S}\right)=\operatorname{ker}\left(\phi_{K / k, S}\right) \otimes \mathbb{Z}_{p}
$$

For an infinite procyclic extension $K / k$, let

$$
\begin{aligned}
\operatorname{ker}\left(\bar{\phi}_{K / k, S}\right) & :=\bigcap_{L} \operatorname{ker}\left(\bar{\phi}_{L / k, S}\right), \\
\left(U_{k}(S) \otimes \mathbb{Z}_{p}\right)^{\text {univ }} & :=\bigcap_{L} N_{L / k}\left(U_{L}(S) \otimes \mathbb{Z}_{p}\right)
\end{aligned}
$$

for all finite cyclic subextension $L$ of $K / k$. Then (HNT) for $U_{k}(S)$ implies that

$$
N_{K / k}\left(U_{K}(S) \otimes \mathbb{Z}_{p}\right) \subset \operatorname{ker}\left(\bar{\phi}_{K / k, S}\right) \subset\left(U_{k}(S) \cap N_{K / k} K^{\times}\right) \otimes \mathbb{Z}_{p}
$$

Definition 3.3. For an infinite procyclic extension $K / k$, if

$$
\operatorname{rank}_{\mathbb{Z}_{p}}\left(\operatorname{ker}\left(\bar{\phi}_{K / k, S}\right)\right)=\operatorname{rank}_{\mathbb{Z}_{p}}\left(\left(U_{k}(S) \otimes \mathbb{Z}_{p}\right)^{\text {univ }}\right),
$$

then we say that $U_{k}(S) \otimes \mathbb{Z}_{p}$ satisfies (HNT) over $K / k$. 
For a $\mathbb{Z}_{p}$-extension $k_{\infty} / k$ and for the set $S$ of primes lying over $p$, we write $\phi_{n}:=\phi_{k_{n} / k, S}$ for the map

$$
\phi_{n}: U_{k}(p) \longrightarrow \prod_{v \mid p} \widehat{H}^{0}\left(G_{k_{n, w} / k_{v}}, k_{n, w}^{\times}\right) \cong \prod_{v \mid p} k_{v}^{\times} / N_{k_{n, w} / k_{v}} k_{n, w}^{\times}
$$

and write

$$
\operatorname{ker}\left(\phi_{\infty}\right):=\operatorname{ker}\left(\phi_{k_{\infty}, S}\right)=\bigcap_{n \geq 0} \operatorname{ker}\left(\phi_{n}\right)
$$

Similarly, we write $\bar{\phi}_{n}:=\phi_{n} \otimes 1$ for the map

$$
\bar{\phi}_{n}: U_{k}(p) \otimes \mathbb{Z}_{p} \longrightarrow \prod_{v \mid p} \widehat{H}^{0}\left(G_{k_{n, w} / k_{v}}, k_{n, w}^{\times}\right) \cong \prod_{v \mid p} k_{v}^{\times} / N_{k_{n, w} / k_{v}} k_{n, w}^{\times}
$$

and write

$$
\operatorname{ker}\left(\bar{\phi}_{\infty}\right):=\operatorname{ker}\left(\bar{\phi}_{k_{\infty}, S}\right)=\bigcap_{n \geq 0} \operatorname{ker}\left(\bar{\phi}_{n}\right)
$$

Remarks. 1. It is well known that the generalized Gross conjecture for $(k, p)$ and Hasse's norm theorem(HNT) for $\mathcal{U}_{k}(p)$ over $k_{\infty}^{\mathrm{cyc}} / k$ are equivalent (cf. [9], [18] and [24]). All proofs use the Kuz'min's result on the invariant of the Tate module of $k$. In fact, independent of this, one can prove it in a more simple and direct way not using the structure of Tate module but using cohomology of the $p$-idele class group of $k$.

2. Notice that in general the first equality in the following equation does not hold

$$
\begin{aligned}
\operatorname{rank}_{\mathbb{Z}_{p}}\left(\operatorname{ker}\left(\bar{\phi}_{\infty}\right)\right) & =\operatorname{rank}_{\mathbb{Z}_{p}}\left(\operatorname{ker}\left(\phi_{\infty}\right) \otimes \mathbb{Z}_{p}\right) \\
& =\operatorname{rank}_{\mathbb{Z}_{p}}\left(U_{k}(p)^{\text {univ }} \otimes \mathbb{Z}_{p}\right)
\end{aligned}
$$

For an abelian field $k$, since the conjectures of Leopoldt and Gross are true, we can apply the remark after Theorem 1.2. Then when $k$ is abelian and $k_{\infty}=k_{\infty}^{\text {cyc }}$, a counterexample to the above equality can be found easily by computing the ranks using Theorem 1.2, and Theorem 2 of [2], i.e.,

$$
\begin{aligned}
r_{1}+r_{2}=\operatorname{rank}_{\mathbb{Z}_{p}}\left(\operatorname{ker}\left(\bar{\phi}_{\infty}\right)\right) & >r_{2}-r^{\prime}+1=\operatorname{rank}_{\mathbb{Z}}\left(k^{\times}\right)^{\text {univ }} \\
& \geq \operatorname{rank}_{\mathbb{Z}}\left(U_{k}(p)^{\text {univ }}\right)=\operatorname{rank}_{\mathbb{Z}}\left(\operatorname{ker}\left(\phi_{\infty}\right)\right)
\end{aligned}
$$

where $r^{\prime}=r$ or $r / 2$ according as the decomposition field at $p$ is real or imaginary. 


\section{2 .}

In this subsection, we will recall Hilbert's theorem 90 for compact submodules over a $\mathbb{Z}_{p}$-extension $k_{\infty}=\bigcup_{n \geq 0} k_{n}$ of $k$. Let $A_{n}$ be a compact $\mathbb{Z}_{p}\left[G\left(k_{n} / k\right)\right]$-module with the norm maps $N_{m, n}: A_{m} \rightarrow A_{n}$ for all $m \geq n$. If we define $A_{\infty}^{(n)}:=\lim _{s \geq n} A_{s}$ to be the inverse limits of $\left\{A_{s}\right\}_{s \geq n}$ with respect to the norm maps $N_{s, n}$, then $A_{\infty}^{(n)}$ is a $\Lambda:=\lim _{s \geq n} \mathbb{Z}_{p}\left[G\left(k_{s} / k_{n}\right)\right]$-module.

Since $A_{n}$ is compact, we have $A_{n}^{\text {univ }}=A_{n}^{\text {comp }}$ and hence we have

$$
A_{\infty}^{(m)}=\underbrace{}_{n \geq m} \lim _{n} A_{n}=\underbrace{}_{n \geq m} \lim _{n}^{\text {univ }}=\lim _{n \geq m} A_{n}^{\text {comp }}
$$

As in the introduction, we say that $A_{k}$ satisfy Hilbert's theorem 90 over $k_{\infty} / k$ if the following isomorphisms hold for all $n \geq 0$,

$$
\left(A_{\infty}\right)_{\Gamma} \cong A_{k}^{\text {univ }}
$$

where $\left(A_{\infty}\right)_{\Gamma}=A_{\infty} /(\gamma-1) A_{\infty}$.

Note that Hilbert's theorem 90 for each finite level of compact group $A_{n}$ will show that

$$
\frac{A_{\infty}^{(n)}}{\varlimsup_{s \geq n}\left(\left(\gamma^{p^{n}}-1\right) A_{s}\right)} \cong A_{n}^{\text {univ }}
$$

In fact, by taking inverse limits in the following commutative diagram

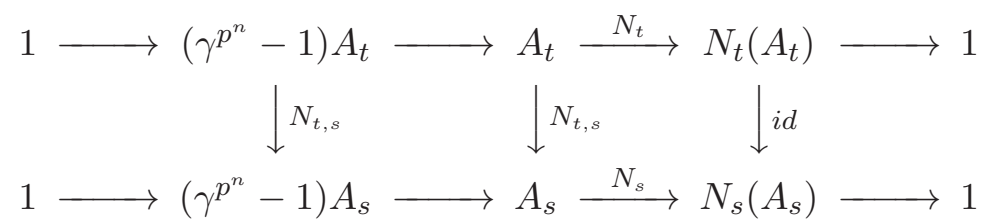

we have the exact sequence

$$
1 \longrightarrow \underbrace{\lim }_{s \geq n}\left(\left(\gamma^{p^{n}}-1\right) A_{s}\right) \longrightarrow A_{\infty}^{(n)} \longrightarrow \bigcap_{s \geq n} N_{s}\left(A_{s}\right)=A_{n}^{\text {univ }} \longrightarrow 1
$$

where inverse limit is exact over compact groups.

Since we have the natural inclusion

$$
\left(\gamma^{p^{n}}-1\right) A_{\infty}^{(n)} \subset \lim _{s \geq n}\left(\left(\gamma^{p^{n}}-1\right) A_{s}\right)
$$


Hilbert's theorem 90 for $A_{n}$ over $k_{\infty} / k_{n}$ for all $n \geq 0$ will be satisfied if Hilbert's theorem 90 for $A_{n}$ is satisfied for all $n \geq 0$, and if the above inclusion becomes equality

$$
\left(\gamma^{p^{n}}-1\right) A_{\infty}^{(n)}=\lim _{s \geq n}\left(\left(\gamma^{p^{n}}-1\right) A_{s}\right)
$$

Notice that the equality above is neither obvious nor easy to determine in general settings. We will show that the modules studied here satisfy the equality.

Let $B_{n}$ be a compact $\mathbb{Z}_{p}\left[G\left(k_{n} / k\right)\right]$-module with norm maps defined as above and let $f_{n}: A_{n} \rightarrow B_{n}$ be the Galois equivariant map so that the following diagram is commutative

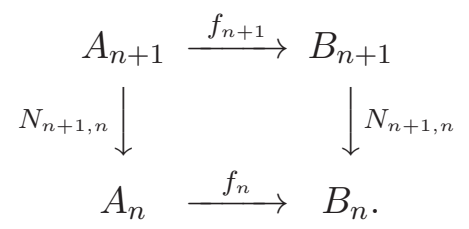

Note that if we further assume that there is a $\Lambda$-module isomorphism

$$
A_{\infty} \cong B_{\infty}
$$

such that $A_{n}$ and $B_{n}$ also satisfy Hilbert's theorem 90 over $k_{\infty} / k$ for all $n \geq 0$, then we have $\mathbb{Z}_{p}\left[G\left(k_{n} / k\right)\right]$-module isomorphism

$$
A_{n}^{\text {univ }} \cong\left(A_{\infty}\right)_{\Gamma_{n}} \cong\left(B_{\infty}\right)_{\Gamma_{n}} \cong B_{n}^{\text {univ }} \text { for all } n \geq 0
$$

For the global $p$-units $U_{n}(p)$ of $k_{n}$, let $A_{n}=\mathcal{U}_{n}(p)=U_{n}(p) \otimes \mathbb{Z}_{p}$ and let $f_{n}=\bar{\Delta}_{n}$ be the map

$$
\bar{\Delta}_{n}: \mathcal{U}_{n}(p) \longrightarrow \prod_{v \mid p} \widehat{k}_{n, v}^{\times} .
$$

This is the $\mathbb{Z}_{p}$-linear extension of the diagonal embedding

$$
\Delta_{n}: U_{n}(p) \longrightarrow \prod_{v \mid p} \widehat{k}_{n, v}^{\times}, \quad \alpha \mapsto(\alpha, \ldots, \alpha)
$$

where $\widehat{k}_{n, v}^{\times}=\lim _{n} k_{n, v}^{\times} / k_{n, v}^{\times p^{n}}$ denotes the $p$-adic completion of the local field $k_{n, v}^{\times}$. Let $B_{n}=\bar{U}_{n}(p):=\bar{\Delta}_{n}\left(\mathcal{U}_{n}(p)\right)$ be the image. Then $\bar{U}_{n}(p)$ is the topological closure of $U_{n}(p)$ under $\Delta_{n}$ in $\prod_{v \mid p} \widehat{k}_{n, v}^{\times}$. 
We have the commutative diagram

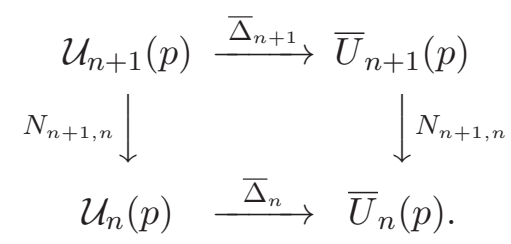

Write

$$
\mathcal{U}_{\infty}^{(n)}:=\underbrace{\lim }_{s \geq n} \mathcal{U}_{n}(p), \quad \bar{U}_{\infty}^{(n)}:={\underset{\varliminf}{s \geq n}}_{\lim _{s}} \bar{U}_{s}(p), \quad \bar{\Delta}^{(n)}:=\prod_{s \geq n} \bar{\Delta}_{s}
$$

and

$$
\mathcal{U}_{\infty}:=\mathcal{U}_{\infty}^{(0)}, \quad \bar{U}_{\infty}:=\bar{U}_{\infty}^{(0)}, \quad \bar{\Delta}:=\bar{\Delta}^{(0)}
$$

The isomorphism of two inverse limits above is known as the weak Leopoldt conjecture for $k_{\infty} / k$ and $p$,

$$
\bar{\Delta}: \mathcal{U}_{\infty} \cong \bar{U}_{\infty}
$$

It is well known that the weak Leopoldt conjecture is true for the cyclotomic $\mathbb{Z}_{p}$-extension $k_{\infty}^{\mathrm{cyc}} / k$ for any number field $k$ and $p$ (cf. $\S 3$ of Ch 10 of [22]).

\section{3.}

In this subsection, we show that the affirmation of the generalized Gross conjecture for $\left(k_{\infty}^{\mathrm{cyc}}, p\right)$ and Hilbert's theorem 90 for $\overline{\Delta\left(U_{n}(p)\right)}$ over $k_{\infty}^{\text {cyc }} / k_{n}$ for all $n \geq 0$ implies the Leopoldt conjecture for $\left(k_{\infty}^{\text {cyc }}, p\right)$. We start with the basic lemmas without proofs which are well known.

Lemma 3.4. $\mathcal{U}_{k}(p)^{\mathrm{univ}}=\mathcal{U}_{k}(p)^{\mathrm{comp}}$.

For the local field $k_{v}$ which is the completion of $k$ at a finite place $v$, let $k_{v, \infty}$ be the corresponding $\mathbb{Z}_{p}$-extension of $k_{v}$. By the definition, we have $k_{v}^{\text {comp }}=\pi\left(\lim _{n} k_{v, n}^{\times}\right)$where $k_{v, n}$ is the subfield of $k_{v, \infty}$ of degree $p^{n}$ over $k_{v}$. Write $k^{\text {toc }}=k^{\times} \bigcap_{v} k_{v}^{\text {comp }}$ for the set of elements which are locally norm 
comparable at all finite places of $k$

$$
\begin{aligned}
& k^{\text {loc }}=\left\{\alpha \in k^{\times} \mid\right. \text {there is }\left(\alpha_{v, n}\right) \in{\underset{\lim }{n}}_{n} k_{v, n} \\
&\text { such that } \left.\alpha_{v, 0}=\alpha \text { for all finite places } v\right\} .
\end{aligned}
$$

By Hasse's local-global norm theorem, $k^{\text {loc }}$ is equal to the group $k^{\text {univ }}$ of the universal norms of $k^{\times}$. Since it is well known that $k^{\text {univ }}$ is contained in the group of $p$-units (see Proposition 2.1 of [2]), we obtain the following lemma.

Lemma 3.5. $k^{\text {loc }}=k^{\text {univ }}=U_{k}(p) \bigcap_{n \geq 0} N_{n} k_{n}^{\times}$.

Let $L$ be the maximal abelian unramified $p$-extension of $k_{\infty}$ where all primes dividing $p$ split completely. Then, obviously,

$$
G\left(L / k_{\infty}\right) \cong T_{p}(k)
$$

and $L$ is Galois over $k$ with the following exact sequence

$$
1 \longrightarrow G\left(L / k_{\infty}\right) \longrightarrow G(L / k) \longrightarrow \Gamma \longrightarrow 1 .
$$

Let $L_{0}$ denote the maximal abelian subextension of $L$ over $k$. Thus we have

$$
G\left(L_{0} / k_{\infty}\right) \cong T_{p}(k)_{\Gamma}
$$

and the exact sequence

$$
1 \longrightarrow G\left(L_{0} / k_{\infty}\right) \longrightarrow G\left(L_{0} / k\right) \longrightarrow \Gamma \longrightarrow 1 .
$$

Let $F_{0}$ denote the maximal abelian extension of $k$ containing $k_{\infty}$ and unramified outside $p$, such that $F / k_{\infty}$ splits completely at all primes dividing $p$. Let $H$ denote the maximal unramified abelian extension of $k$ such that all primes dividing $p$ split completely and $K$ the maximal $p$-extension of $k$ in $H$.

Let $S_{v}$ denote the kernel of the Artin map $k_{v}^{\times} \longrightarrow G\left(k_{\infty} / k\right)$. Then, the class field theory says that $S_{v}$ is the group of universal norm elements of $k_{v}^{\times}$ over $k_{\infty} / k$. By the class field theory, we have the following isomorphism

$$
G\left(F_{0} / k\right) \cong J_{k} / A
$$

where $A=\overline{k^{\times} \prod_{v \nmid p} U_{v} \prod_{v \mid p} S_{v}}$ is the topological p-adic closure of $k^{\times} \prod_{v \nmid p} U_{v}$. $\prod_{v \mid p} S_{v}$ (see the last line of page 455 in [6] or the equation 3.4 of page 280 
in [18]). There is exact sequence for the $G(H / k)$

$$
1 \longrightarrow k^{\times} \prod_{v \nmid p} U_{v} \prod_{v \mid p} k_{v}^{\times} / A \longrightarrow J_{k} / A \longrightarrow G(H / k) \longrightarrow 1
$$

induced from $1 \longrightarrow k^{\times} \prod_{v \nmid p} U_{v} \prod_{v \mid p} k_{v}^{\times} \longrightarrow J_{k} \longrightarrow G(H / k) \longrightarrow 1$.

Let $V_{p}^{n}$ be the subgroup of $\prod_{v \mid p} U_{v}$ which are $\equiv 1 \bmod p^{n}$

$$
V_{p}^{n}=\left\{a \in \prod_{v \mid p} U_{v} \mid a \equiv 1 \bmod p^{n}\right\}
$$

Then the groups $V_{p}^{n} k^{\times} \prod_{v \nmid p} U_{v} \prod_{v \mid p} S_{v}$ forms a fundamental system of neighborhoods of the topological $p$-adic closure $A$ of $k^{\times} \prod_{v \nmid p} U_{v} \prod_{v \mid p} S_{v}$ and its closure is given as follows (see the proof of Theorem 7.8 of [21])

$$
\overline{k^{\times} \prod_{v \nmid p} U_{v} \prod_{v \mid p} S_{v}}=\bigcap_{n}\left(V_{p}^{n} k^{\times} \prod_{v \nmid p} U_{v} \prod_{v \mid p} S_{v}\right) .
$$

The first term $G\left(F_{0} / H\right) \cong k^{\times} \prod_{v \nmid p} U_{v} \prod_{v \mid p} k_{v}^{\times} / \overline{k^{\times} \prod_{v \nmid p} U_{v} \prod_{v \mid p} S_{v}}$ of the short exact sequence above leads to the following exact sequence (see p. 456 of [6] or page 280 of [18])

$$
1 \longrightarrow X \longrightarrow \prod_{v \mid p}\left(k_{v}^{\times} / S_{v}\right) \longrightarrow G\left(F_{0} / H\right) \longrightarrow 1
$$

where

$$
X=\prod_{v \mid p} S_{v} \overline{\delta\left(U_{k}(p)\right)} / \prod_{v \mid p} S_{v}
$$

denotes the topological $p$-adic closure of the image $U_{k}(p) \longrightarrow \prod_{v \mid p}\left(k_{v}^{\times} / S_{v}\right)$.

In fact, we see that the first term $k^{\times} \prod_{v \nmid p} U_{v} \prod_{v \mid p} k_{v}^{\times} / \overline{k^{\times} \prod_{v \nmid p} U_{v} \prod_{v \mid p} S_{v}}$ is isomorphic to

$$
\begin{aligned}
\prod_{v \mid p} k_{v}^{\times} / \prod_{v \mid p} k_{v}^{\times} \cap \overline{k^{\times} \prod_{v \nmid p} U_{v} \prod_{v \mid p} S_{v}} & \left.=\prod_{v \mid p} k_{v}^{\times} / \prod_{v \mid p} k_{v}^{\times} \cap \bigcap V_{p}^{n} k^{\times} \prod_{v \nmid p} U_{v} \prod_{v \mid p} S_{v}\right) \\
& =\prod_{v \mid p} k_{v}^{\times} / \prod_{v \mid p} S_{v}\left(\prod_{v \mid p} k_{v}^{\times} \cap \bigcap V_{p}^{n} k^{\times} \prod_{v \nmid p} U_{v}\right)
\end{aligned}
$$


Since

$$
\begin{aligned}
\prod_{v \mid p} S_{v}\left(\bigcap V_{p}^{n}\left(\prod_{v \mid p} k_{v}^{\times} \cap k^{\times} \prod_{v \nmid p} U_{v}\right)\right) & =\prod_{v \mid p} S_{v}\left(\bigcap V_{p}^{n}\left(\delta\left(U_{k}(p)\right)\right)\right) \\
& =\prod_{v \mid p} S_{v} \overline{\delta\left(U_{k}(p)\right)}
\end{aligned}
$$

the first term above is isomorphic to

$$
\prod_{v \mid p}\left(k_{v}^{\times} / S_{v} \overline{\delta\left(U_{k}(p)\right)}\right) \cong \prod_{v \mid p}\left(k_{v}^{\times} / S_{v}\right) / \prod_{v \mid p}\left(S_{v} \overline{\delta\left(U_{k}(p)\right)} / S_{v}\right) .
$$

From the identities above, the Artin map induces the exact sequence above. Moreover, it was shown from this that $G\left(F_{0} / H\right)$ is a $p$-group (see p. 456 of $[6])$, i.e.,

$$
G\left(F_{0} / H\right) \cong G\left(L_{0} / K\right)
$$

Let $r$ be the number of primes of $k$ dividing $p$. By the assumption of Gross conjecture, the $\mathbb{Z}_{p}$-rank of $G\left(F_{0} / H\right)$ is equal to one since the $\mathbb{Z}_{p}$-rank of $G\left(L_{0} / k_{\infty}\right) \cong T_{p}(k)_{\Gamma}$ is zero. Then, since $G(K / k)$ is finite, the rank of the topological $p$-adic closure $X(p)$ of $X$ is given by

$$
\operatorname{rank}(X(p))=r-1 .
$$

It follows from the isomorphism

$$
X=\prod_{v \mid p} S_{v} \overline{\delta\left(U_{k}(p)\right)} / \prod_{v \mid p} S_{v} \cong \overline{\delta\left(U_{k}(p)\right)} / \overline{\delta\left(U_{k}(p)\right)} \cap \prod_{v \mid p} S_{v}
$$

that there exists a short exact sequence for $\overline{\delta\left(U_{k}(p)\right)} \cap \prod_{v \mid p} S_{v}$

$$
1 \longrightarrow \overline{\delta\left(U_{k}(p)\right)} \cap \prod_{v \mid p} S_{v} \longrightarrow \overline{\delta\left(U_{k}(p)\right)} \longrightarrow X \longrightarrow 1 .
$$

Note that the topological $p$-adic closure $\overline{\delta\left(U_{k}(p)\right)} \wedge$ of $\overline{\delta\left(U_{k}(p)\right)}$ in $\prod_{v \mid p} \widehat{k}_{v}^{\times}$ is equal to $\overline{\Delta\left(U_{k}(p)\right)}$,

$$
\overline{\delta\left(U_{k}(p)\right)} \wedge=\overline{\Delta\left(U_{k}(p)\right)} .
$$

We can compare the $\mathbb{Z}_{p}$-ranks of the topological $p$-adic closures of each terms above

$$
\operatorname{rank}\left(\overline{\Delta\left(U_{k}(p)\right)}\right)=\operatorname{rank}(\Omega)+\operatorname{rank}(X(p))
$$

where $\Omega$ denotes the topological $p$-adic closure of $\overline{\delta\left(U_{k}(p)\right)} \cap \prod_{v \mid p} S_{v}$. 
We may assume that $k_{\infty} / k$ is totally ramified at primes dividing $p$ since the Leopoldt conjecture descends to a subfield (see Corollary 10.3.11 of [22]), i.e., the validity of the Leopoldt conjecture for a number field $K$ implies the validity of the Leopoldt conjecture for any subfield of $K$. We will use the same notation $v$ for the prime of $k_{n}$ lying over $p$ as that of $k$.

It follows from $\S 3.1$ that

$$
U_{k}(p) \cap \prod_{v \mid p} N_{n_{v}} k_{n, v}^{\times}=\operatorname{ker}\left(\phi_{n}\right)
$$

and that $\delta$ induces $\tilde{\delta}$

$$
1 \longrightarrow U_{k}(p) / \operatorname{ker}\left(\phi_{n}\right) \stackrel{\tilde{\delta}}{\longrightarrow} \prod_{v \mid p} k_{v}^{\times} / N_{n_{v}} k_{n, v}^{\times}
$$

where $k_{n, v}$ denotes the completion of $k_{n}$ at a prime dividing $v$ and $N_{n_{v}}$ denotes the norm map from $k_{n, v}$ to $k_{v}$. By taking topological closure inside $\prod_{v \mid p} N_{n_{v}} k_{n, v}^{\times}$to the above identity, we have

$$
\overline{\left(\delta\left(U_{k}(p) \cap \prod_{v \mid p} N_{n_{v}} k_{n, v}^{\times}\right)\right.}=\overline{\delta\left(U_{k}(p)\right)} \cap \prod_{v \mid p} N_{n_{v}} k_{n, v}^{\times}=\overline{\delta\left(\operatorname{ker}\left(\phi_{n}\right)\right)} .
$$

This leads to

$$
\begin{aligned}
\overline{\delta\left(U_{k}(p)\right)} \cap \prod_{v \mid p} S_{v} & \left.=\bigcap_{n} \overline{\left(\delta\left(U_{k}(p)\right)\right.} \cap \prod_{v \mid p} N_{n_{v}} k_{n, v}^{\times}\right) \\
& =\bigcap_{n} \overline{\delta\left(\operatorname{ker}\left(\phi_{n}\right)\right)}
\end{aligned}
$$

By the generalized Gross conjecture, it follows that

$$
\left(\overline{\delta\left(\operatorname{ker}\left(\phi_{n}\right)\right)}: \overline{\delta\left(N_{n}\left(U_{n}\right)\right)}\right)<\left(\delta\left(\operatorname{ker}\left(\phi_{n}\right)\right): \delta\left(N_{n}\left(U_{n}\right)\right)\right)<\infty
$$

independently of $n \geq 0$ and that

$$
\begin{aligned}
\operatorname{rank} \overline{\delta\left(U_{k}(p)\right)} \cap \prod_{v \mid p} S_{v} & =\operatorname{rank} \bigcap_{n} \overline{\delta\left(N_{n}\left(U_{n}\right)\right)}=\operatorname{rank} \bigcap_{n} N_{n}\left(\overline{\delta\left(U_{n}\right)}\right) \\
& =\operatorname{rank} \overline{\delta\left(U_{k}(p)\right)^{\text {univ }}}
\end{aligned}
$$


By taking closures, the inclusion above results in

$$
\begin{aligned}
\operatorname{rank} \Omega=\operatorname{rank}\left(\overline{\delta\left(U_{k}(p)\right)} \cap \prod_{v \mid p} S_{v}\right)^{\wedge} & =\operatorname{rank}\left({\overline{\delta\left(U_{k}(p)\right)}}^{\text {univ }}\right)^{\wedge} \\
& =\operatorname{rank}{\overline{\Delta\left(U_{k}(p)\right)}}^{\text {univ }} .
\end{aligned}
$$

Here, the identity $\left({\overline{\delta\left(U_{k}(p)\right)}}^{\text {univ }}\right)^{\wedge}={\overline{\Delta\left(U_{k}(p)\right)}}^{\text {univ }}$ follows from the the following argument using the assumption that $k_{\infty} / k$ is totally ramified at all primes dividing $p$.

By the local Kronecker-Weber theorem, the intermediate field $k_{v, n} / k_{v}$ is contained in a composite field $k_{v, n}^{(f)} L_{m}$ of the field $L_{m}$ of the $\pi^{m}$-division points of Lubin-Tate extensions and the unramified extension $k_{v, n}^{(f)}$ of degree $f$ (see the proof of Corollary 7.7 of [21]).

By taking the ramification indices and linear disjointness over $k_{v}$ of $L_{m}$ and $k_{v, n}^{(f)}$ into account, we see that $k_{v, n}$ is contained in $L_{m}$. Since the prime element $\pi_{v}$ is a norm element of a prime element $\lambda_{m}$ of $L_{m}$ (see Theorem 7.4 of [21]), $\pi_{v}$ is the norm of the prime element $N_{L_{m} / k_{v, n}} \lambda_{m}$ of $k_{v, n}$. It follows that any prime element $\pi_{v}$ dividing $p$ is a universal norm element over $k_{v, \infty} / k_{v}$. Since the $p$-adic closure $\widehat{k}_{v}$ of $k_{v}^{\times}=\pi_{v}^{\mathbb{Z}} \oplus U_{v}$ is given by

$$
\widehat{k}_{v}=\pi_{v}^{\mathbb{Z}_{p}} \oplus U_{v}^{1}
$$

where $U_{v}^{1}$ is the principal unit at $v$, we have

$$
\left(\widehat{k}_{v}\right)^{\text {univ }}=\pi_{v}^{\mathbb{Z}_{p}} \oplus\left(U_{v}^{1}\right)^{\text {univ }}
$$

using universal norm property of $\pi_{v}$. Since the norm map is continuous, it follows that $\left(U_{v}^{1}\right)^{\text {univ }}$ is a closed subgroup of $U_{v}^{1}=\widehat{U_{v}}$ and that $\left(\left(U_{v}^{1}\right)^{\text {univ }}\right)^{\wedge}=$ $\left(U_{v}^{1}\right)^{\text {univ }}$, i.e.,

$$
\widehat{U_{v}^{\text {univ }}}=\left(\mu_{q} \times\left(U_{v}^{1}\right)^{\text {univ }}\right)^{\wedge}=\left(U_{v}^{1}\right)^{\text {univ }}=\widehat{U}_{v}^{\text {univ }}
$$

where $U_{v}=\mu_{q} \times U_{v}^{1}$ for $q=\#\left(U_{v} / U_{v}^{1}\right)$. It follows that the $p$-adic closure $\widehat{\left(k_{v}^{\text {univ }}\right)}$ of $k_{v}^{\text {univ }}$ is given by

$$
\widehat{\left(k_{v}^{\text {univ }}\right)}=\left(\pi_{v}^{\mathbb{Z}} \oplus U_{v}^{\text {univ }}\right)^{\wedge}=\pi_{v}^{\mathbb{Z}_{p}} \oplus\left(U_{v}^{1}\right)^{\text {univ }}=\left(\widehat{k}_{v}\right)^{\text {univ }} .
$$

Note that the difference between $\overline{\delta\left(U_{k}(p)\right)} \subset \prod k_{v}^{\times}$and $\overline{\Delta\left(U_{k}(p)\right)} \subset \prod \widehat{k}_{v}^{\times}$ occurs only in the cyclic groups generated by each prime $\pi_{v}$ dividing $p$, one 
$\pi_{v}^{\mathbb{Z}}$ and the other $\pi_{v}^{\mathbb{Z}_{p}}$ which are, by the assumption, already norm comparable, and its torsion subgroups in unit-parts which are also norm comparable. Hence the closure $\left({\overline{\delta\left(U_{k}(p)\right)}}^{\text {univ }}\right)^{\wedge}$ is equal to ${\overline{\Delta\left(U_{k}(p)\right)}}^{\text {univ }}$ as was claimed. It follows from Theorems 3.6 and 3.7 of $\S 3.4$ that

$$
\operatorname{rank} \mathcal{U}_{k}(p)^{\mathrm{univ}}=\operatorname{rank}\left(U_{k}^{\prime}(p) \otimes \mathbb{Z}_{p}\right)^{\mathrm{univ}}=\operatorname{rank}\left(\mathcal{U}_{\infty}^{\prime}\right)_{\Gamma}=r_{1}+r_{2}
$$

Since the weak Leopoldt conjecture is true for the cyclotomic $\mathbb{Z}_{p}$-extension of any number field, we have (see Corollary 10.3.24 and Theorem 10.3.25 of $[22])$

$$
\overline{\Delta\left(U_{\infty}(p)\right)}:=\lim _{\longleftarrow} \overline{\Delta\left(U_{n}(p)\right)} \cong \lim _{\longleftarrow}\left(\mathcal{U}_{n}(p)\right)=\mathcal{U}_{\infty} .
$$

The above isomorphism and Hilbert's theorem 90 for $\overline{\Delta\left(U_{k}(p)\right)}$ lead to the following lower bound of $\operatorname{rank}(\Omega)$

$$
\begin{aligned}
\operatorname{rank}(\Omega) \geq \operatorname{rank}\left(\overline{\Delta\left(U_{k}(p)\right)}{ }^{\text {univ }}\right) & =\operatorname{rank}\left({\overline{\Delta\left(U_{\infty}(p)\right)_{\Gamma}}}\right. \\
& =\operatorname{rank}\left(\mathcal{U}_{\infty}\right)_{\Gamma} \\
& =\operatorname{rank} \mathcal{U}_{k}(p)^{\text {univ }} \\
& =r_{1}+r_{2} .
\end{aligned}
$$

Hence it results in the Leopoldt conjecture

$$
\begin{aligned}
\operatorname{rank}\left(\overline{\Delta\left(U_{k}(p)\right)}\right) & =\operatorname{rank}(X(p))+\operatorname{rank}(\Omega) \\
& \geq r-1+r_{1}+r_{2} .
\end{aligned}
$$

This shows that if we assume that $k_{\infty} / k$ is totally ramified, then the generalized Gross conjecture and Hilbert's theorem 90 imply the Leopoldt conjecture. Since for an arbitrary number field $k$, there exists a sufficiently large $n$ such that $k_{\infty} / k_{n}$ is totally ramified at all primes dividing $p$ (cf. $\S \S 3.4$ and 6.2 of [12]), we have shown that for such $n$, the assumption implies the validity of the Leopoldt conjecture for $k_{n}$. Since the Leoplodt conjecture descends to a subfield, we obtain the validity of the Leopoldt conjecture for $k$ from $k_{n}$. This completes the proof.

\section{4.}

In this subsection, we will show that the Leopoldt conjecture for $(k, p)$ implies Hilbert's theorem 90 for $\overline{\Delta\left(U_{k}(p)\right)}$ over $k_{\infty}^{\text {cyc }} / k$. 
For the $p$-units $U_{n}(p)$ of $k_{n}$, let $U_{n}^{\prime}(p)=U_{n}(p) / \mu\left(U_{n}(p)\right)$ be the quotient of $U_{n}(p)$ by its torsion $\mu\left(U_{n}(p)\right)$, i.e., the $\mathbb{Z}$-free part of $U_{n}(p)$ and let

$$
\mathcal{U}_{\infty}^{\prime}=\lim _{\longleftarrow}\left(U_{n}^{\prime}(p) \otimes \mathbb{Z}_{p}\right)
$$

denote the inverse limit of $U_{n}^{\prime}(p) \otimes \mathbb{Z}_{p}$ with respect to the norm maps. The rank of $\mathcal{U}_{\infty}^{\prime}$ as a $\Gamma$-module is computed in the following theorem which is Theorem 7.2 of [18]. He proves this over the cyclotomic $\mathbb{Z}_{p}$-extension of $k$. This is reproved in a generalized form by Greither (see Theorem of [9]).

In Theorem 3.7, we give a proof of the rank of $\left(U_{k}^{\prime}(p) \otimes \mathbb{Z}_{p}\right)^{\text {univ }}$ for any $\mathbb{Z}_{p}$-extension of $k$ using a result of Iwasawa together with an argument of compactness and hence different from those of Kuz'min and Greither. Note that the proof of Greither uses the direct limits rather than the inverse limits of ours. Even if it seems to be dual each other, the proof given below we think is more simple and direct.

Theorem 3.6. Let $k$ be a number field and let $r_{1}$ and $r_{2}$ be the number of real and complex places of $k$. Then $\mathcal{U}_{\infty}^{\prime}$ is a free $\Gamma$-module of rank $r_{1}+r_{2}$.

Proof. See the proof of Theorem 7.2 of [18]. Since the weak Leopoldt conjecture holds for the cyclotomic $\mathbb{Z}_{p}$-extension of a number field, we can find another proof from Corollary 10.3.24 and Theorem 11.3.11 of [22].

In fact, the statement $(i i)$ of Theorem 11.3 .11 of loc.cit holds with $S_{\infty}^{\prime}=$ $S^{c d}=\varnothing$ for the cyclotomic $\mathbb{Z}_{p}$-extension over any number field (cf. Remark and Corollary 11.3.12 of loc.cit).

Theorem 3.7. Let $k_{\infty}=\bigcup_{n \geq 0} k_{n}$ be an arbitrary $\mathbb{Z}_{p}$-extension of $k$. Then

$$
\operatorname{rank}\left(\mathcal{U}_{\infty}^{\prime}\right)_{\Gamma}=\operatorname{rank}\left(U_{k}^{\prime}(p) \otimes \mathbb{Z}_{p}\right)^{\text {univ }}=r_{1}+r_{2}
$$

Proof. For a $\mathbb{Z}\left[G\left(k_{n} / k\right)\right]$-module $M$, we have

$$
1 \longrightarrow H^{-1}\left(G\left(k_{n} / k\right), M\right) \longrightarrow M /(\gamma-1) M \stackrel{N_{n}}{\longrightarrow} N_{n}(M) \longrightarrow 1 .
$$

Since the $\mathbb{Z}$-module $\mathbb{Z}_{p}$ is flat (see (iii) of Theorem 3 of $\S 3.4$ of [4]), we have

$$
\begin{aligned}
N_{n}(M) \otimes_{\mathbb{Z}} \mathbb{Z}_{p} & =N_{n}\left(M \otimes_{\mathbb{Z}} \mathbb{Z}_{p}\right) \\
(M /(\gamma-1) M) \otimes \mathbb{Z}_{p} & =\left(M \otimes \mathbb{Z}_{p}\right) /(\gamma-1)\left(M \otimes \mathbb{Z}_{p}\right) .
\end{aligned}
$$


By tensoring $\mathbb{Z}_{p}$, the exact sequence leads to

$$
\begin{aligned}
1 & \longrightarrow H^{-1}\left(G\left(k_{n} / k\right), M\right) \otimes \mathbb{Z}_{p} \\
& \longrightarrow M \otimes \mathbb{Z}_{p} /(\gamma-1)\left(M \otimes \mathbb{Z}_{p}\right) \stackrel{N_{n} \otimes 1}{\longrightarrow} N_{n}\left(M \otimes \mathbb{Z}_{p}\right) \longrightarrow 1
\end{aligned}
$$

This shows the following isomorphism

$$
H^{-1}\left(G\left(k_{n} / k\right), M\right) \otimes \mathbb{Z}_{p} \cong H^{-1}\left(G\left(k_{n} / k\right), M \otimes \mathbb{Z}_{p}\right)
$$

By putting $M=k_{n}^{\times}$and using Hilbert's theorem 90 for $k_{n}^{\times}$, the above isomorphism leads to

$$
\operatorname{ker}\left(N_{n} \otimes 1\right)=(\gamma-1)\left(k_{n}^{\times} \otimes \mathbb{Z}_{p}\right)
$$

Hence we have the following commutative diagram

$$
\begin{gathered}
1 \longrightarrow\left((\gamma-1)\left(k_{n+1}^{\times} \otimes \mathbb{Z}_{p}\right)\right) \cap \mathcal{U}_{n+1}(p)^{\text {univ }} \longrightarrow \mathcal{U}_{n+1}(p)^{\text {univ }} \\
1 \longrightarrow\left((\gamma-1)\left(k_{n}^{\times} \otimes \mathbb{Z}_{p}\right)\right) \cap \mathcal{U}_{n}(p)^{\text {univ }} \longrightarrow \mathcal{L}^{N_{n+1, n}} \\
\stackrel{N_{n+1} \otimes 1}{\longrightarrow} \mathcal{U}_{k}(p)^{\text {univ }} \longrightarrow \mathcal{U}_{n}(p)^{\text {univ }} \\
\stackrel{N^{\text {ud }}}{\longrightarrow} \mathcal{U}_{k}(p)^{\text {univ }} \longrightarrow 1
\end{gathered}
$$

where the surjectiveness of each rows follows from

$$
\mathcal{U}_{n}(p)^{\text {univ }}=\mathcal{U}_{n}(p)^{\mathrm{comp}}
$$

which is Lemma 3.4. Write

$$
V_{n}=\left((\gamma-1)\left(k_{n}^{\times} \otimes \mathbb{Z}_{p}\right)\right) \cap \mathcal{U}_{n}(p)
$$

By taking inverse limits with respect to the norm maps and using exactness of inverse limit over compact groups, we have

$$
1 \longrightarrow{\underset{n}{n}}_{\lim _{n}} V_{n} \longrightarrow \mathcal{U}_{\infty} \longrightarrow \mathcal{U}_{k}(p)^{\text {univ }} \longrightarrow 1 \text {. }
$$

Lemma 3.8. $\lim _{n} V_{n}=(\gamma-1) \mathcal{U}_{\infty}$. 
Proof. To prove Lemma 3.8, we need the following result of Iwasawa.

Lemma 3.9 (Iwasawa). The order $\#\left(H^{1}\left(G\left(k_{n} / k\right), U_{n}(p)\right)\right)$ is bounded independently of $n$.

Proof. This follows immediately from [12]. More precisely, since every primes outside $p$ are unramified over any $\mathbb{Z}_{p}$-extension $k_{\infty} / k$, Proposition 3 of [12] and the five term exact sequence of Hochschild-Serre spectral sequence lead to the proof of Lemma 3.9 (see Corollary 2.4.2 of [22] and Proposition 13.2 of $[25])$.

Notice that from Hilbert's theorem 90,

$$
H^{1}\left(G\left(k_{n} / k\right), U_{n}(p)\right) \cong H^{-1}\left(G\left(k_{n} / k\right), U_{n}(p)\right)=\frac{\left((\gamma-1) k_{n}^{\times}\right) \cap U_{n}(p)}{(\gamma-1) U_{n}(p)}
$$

We claim that for $s \geq n$, the following inclusion map induced from the identity map is injective

$$
\frac{\left((\gamma-1) k_{n}^{\times}\right) \cap U_{n}(p)}{(\gamma-1) U_{n}(p)} \longrightarrow \frac{\left((\gamma-1) k_{s}^{\times}\right) \cap U_{s}(p)}{(\gamma-1) U_{s}(p)} .
$$

For an $(\gamma-1) \alpha \in(\gamma-1) k_{n}^{\times} \cap U_{n}(p)$, let

$$
(\gamma-1) \alpha=(\gamma-1) e
$$

for some $e \in U_{s}(p)$. Then $a \alpha=e \in U_{s}(p) \cap k_{n}^{\times}=U_{n}(p)$ for some $a \in k^{\times}$. Hence

$$
(\gamma-1) \alpha=(\gamma-1)(a \alpha) \in(\gamma-1) U_{n}(p)
$$

which leads to the injection of the natural map induced from the inclusion

$$
1 \longrightarrow \frac{\left((\gamma-1) k_{n}^{\times}\right) \cap U_{n}(p)}{(\gamma-1) U_{n}(p)} \longrightarrow \frac{\left((\gamma-1) k_{s}^{\times}\right) \cap U_{s}(p)}{(\gamma-1) U_{s}(p)} .
$$

By Lemma 3.9, we have the following lemma.

Lemma 3.10. For all sufficiently large $s \geq n \gg 0$, the inclusion map induces an isomorphism

$$
\frac{\left((\gamma-1) k_{n}^{\times}\right) \cap U_{n}(p)}{(\gamma-1) U_{n}(p)} \cong \frac{\left((\gamma-1) k_{s}^{\times}\right) \cap U_{s}(p)}{(\gamma-1) U_{s}(p)}<\infty .
$$


Since $H^{-1}\left(G\left(k_{n} / k\right), U_{n}(p)\right)=(\gamma-1) k_{n}^{\times} \cap U_{n}(p) /(\gamma-1) U_{n}(p)$ is a $p$-group and the norm map $N_{s, n}$ is the $p^{s-n}$ power map for all sufficiently large $s \geq n$ by Lemma 3.10, we have

$$
\lim _{\longleftarrow}\left(\frac{(\gamma-1) k_{n}^{\times} \cap U_{n}(p)}{(\gamma-1) U_{n}(p)}\right)=1 .
$$

This results in the following corollary.

Corollary 3.11. $\underset{\longleftarrow}{\lim }\left((\gamma-1) k_{n}^{\times} \cap U_{n}(p)\right)=\lim _{\longleftarrow}\left((\gamma-1) U_{n}(p)\right)$.

Since $\mathbb{Z}_{p}$ is flat over $\mathbb{Z}$, we have

$$
\begin{aligned}
1 \rightarrow(\gamma-1) U_{n}(p) \otimes \mathbb{Z}_{p} & \longrightarrow\left((\gamma-1) k_{n}^{\times} \cap U_{n}(p)\right) \otimes \mathbb{Z}_{p} \\
& \longrightarrow\left(\frac{(\gamma-1) k_{n}^{\times} \cap U_{n}(p)}{(\gamma-1) U_{n}(p)}\right) \otimes \mathbb{Z}_{p} \rightarrow 1 .
\end{aligned}
$$

By taking inverse limits with respect to the norm maps over compact groups, we have

$$
\begin{aligned}
1 & \rightarrow \underset{\leftarrow}{\lim }\left((\gamma-1) U_{n}(p) \otimes \mathbb{Z}_{p}\right) \rightarrow \underset{\lim }{\longleftarrow}\left(\left((\gamma-1) k_{n}^{\times} \cap U_{n}(p)\right) \otimes \mathbb{Z}_{p}\right) \\
& \rightarrow \lim _{\longleftarrow}\left(\left(\frac{(\gamma-1) k_{n}^{\times} \cap U_{n}(p)}{(\gamma-1) U_{n}(p)}\right) \otimes \mathbb{Z}_{p}\right) \rightarrow 1 .
\end{aligned}
$$

Since $H^{-1}\left(G\left(k_{n} / k\right), U_{n}(p)\right)$ is a $p$-group, it follows from Corollary 3.11 that

$$
\lim _{\longleftarrow}\left(\left(\frac{(\gamma-1) k_{n}^{\times} \cap U_{n}(p)}{(\gamma-1) U_{n}(p)}\right) \otimes \mathbb{Z}_{p}\right)=\lim _{\longleftarrow}\left(\frac{(\gamma-1) k_{n}^{\times} \cap U_{n}(p)}{(\gamma-1) U_{n}(p)}\right)=1
$$

and hence the exact sequence leads to the following isomorphism

$$
\lim _{\longleftarrow} V_{n}=\lim _{\longleftarrow}\left(\left((\gamma-1) k_{n}^{\times} \cap U_{n}(p)\right) \otimes \mathbb{Z}_{p}\right)=\lim _{\longleftarrow}\left((\gamma-1) U_{n}(p) \otimes \mathbb{Z}_{p}\right)
$$

where we used

$$
\left((\gamma-1) k_{n}^{\times} \cap U_{n}(p)\right) \otimes \mathbb{Z}_{p}=\left((\gamma-1) k_{n}^{\times} \otimes \mathbb{Z}_{p}\right) \cap\left(U_{n}(p) \otimes \mathbb{Z}_{p}\right)=V_{n}
$$

since $\mathbb{Z}_{p}$ is a flat over $\mathbb{Z}$ (see Remark 1 of $\S 2.6$, Chapter I of [3]). 
The exact sequence $1 \longrightarrow U_{k}(p) \longrightarrow U_{n}(p) \stackrel{\gamma-1}{\longrightarrow}(\gamma-1) U_{n}(p) \longrightarrow 1$ leads to

$$
1 \longrightarrow U_{k}(p) \otimes \mathbb{Z}_{p} \longrightarrow U_{n}(p) \otimes \mathbb{Z}_{p} \stackrel{\gamma-1}{\longrightarrow}(\gamma-1) U_{n}(p) \otimes \mathbb{Z}_{p} \longrightarrow 1
$$

By taking inverse limits over compact groups, we have

$$
(\gamma-1) \mathcal{U}_{\infty} \cong \lim _{\longleftarrow}\left((\gamma-1) U_{n}(p) \otimes \mathbb{Z}_{p}\right)
$$

We obtain the proof of the claim

$$
\lim _{\longleftarrow} V_{n} \cong(\gamma-1) \mathcal{U}_{\infty}
$$

and thereby complete the proof of Lemma 3.8 .

Lemma 3.8 and Theorem 3.6 complete the proof of Theorem 3.7.

From the weak Leopoldt conjecture for $(k, p)$, Theorems 3.6 and 3.7 lead to

$$
\operatorname{rank}\left(\lim _{\longleftarrow} \overline{\Delta\left(U_{n}(p)\right)}\right)_{\Gamma}=r_{1}+r_{2} \text {. }
$$

It follows from the natural surjective map $\left(\lim _{\longleftarrow} \overline{\Delta\left(U_{n}(p)\right)}\right)_{\Gamma} \rightarrow{\overline{\Delta\left(U_{k}(p)\right)}}^{\text {univ }} \rightarrow$ 1 that ${\overline{\Delta\left(U_{k}(p)\right)}}^{\text {univ }}$ has $\mathbb{Z}_{p}$-rank at most $r_{1}+r_{2}$. From the Leopoldt conjecture for $(k, p), \bar{\Delta}_{k}$ induces an injection $1 \rightarrow\left(\mathcal{U}_{k}(p)\right)^{\text {univ }} \rightarrow{\overline{\Delta\left(U_{k}(p)\right)}}^{\text {univ }}$ which shows from Theorem 3.7 that $\overline{\Delta\left(U_{k}(p)\right)}{ }^{\text {univ }}$ has $\mathbb{Z}_{p}$-rank at least $r_{1}+r_{2}$ and hence exactly $r_{1}+r_{2}$. It follows that

$$
{\overline{\Delta\left(U_{k}(p)\right)}}^{\text {univ }} \cong\left(\mathcal{U}_{k}(p)\right)^{\text {univ }} \cong\left(\mathcal{U}_{\infty}\right)_{\Gamma} \cong\left(\lim _{\longleftarrow} \overline{\Delta\left(U_{n}(p)\right)}\right)_{\Gamma}
$$

which is Hilbert's theorem 90 for $\overline{\Delta\left(U_{k}(p)\right)}$. This completes the proof of Theorem 1.2.

Remark. We notice from the proof of Theorem 1.2 that if $k_{\infty}^{\text {cyc }} / k$ is totally ramified then the affirmation of the Leopoldt conjecture for $(k, p)$ is equivalent to the affirmation of Hilbert's theorem 90 for $\overline{\Delta\left(U_{k}(p)\right)}$ over $k_{\infty}^{\text {cyc }} / k$.

\section{5.}

In this subsection we prove Corollary 1.3 of the introduction and explain special cases of the corollary. We recall Corollary 1.3 of the introduction. 
Corollary 3.12. (i) If $k$ is a totally real field then the affirmation of the Leopoldt conjecture for $\left(k_{\infty}^{\mathrm{cyc}}, p\right)$ is equivalent to the affirmation of the Gross conjecture for $\left(k_{\infty}^{\text {cyc }}, p\right)$ and Hilbert's theorem 90 for $\overline{\Delta\left(U_{n}(p)\right)}$ over $k_{\infty}^{\text {cyc }} / k_{n}$ for all $n \geq 0$.

(ii) If $k$ is a CM-field with a maximal real subfield $k^{+}$then the affirmation of the Leopoldt conjecture for $\left(k_{\infty}^{\mathrm{cyc}}, p\right)$ is equivalent to the affirmation of the Gross conjecture for $\left(k_{\infty}^{+\mathrm{cyc}}, p\right)$ and Hilbert's theorem 90 for $\overline{\Delta\left(U_{k_{n}^{+}}(p)\right)}$ over $k_{\infty}^{+ \text {cyc }} / k_{n}^{+}$for all $n \geq 0$.

Proof. It is well known that the Leopoldt conjecture implies the generalized Gross conjecture when the base field $k$ is totally real. In fact, we immediately recover this from the equations (1) and (2) in the proof of Theorem 1.2 since the Leopoldt conjecture is equivalent to the claim that $k$ has only one $\mathbb{Z}_{p^{-}}$ extension when $k$ is totally real. Hence from Theorem 1.2, we prove the first claim of the corollary.

For a CM-field $k$ with its maximal real subfield $k^{+}$, it is also well known that the affirmation of the Leopoldt conjecture for $(k, p)$ is equivalent to the affirmation of the Leopoldt conjecture for $\left(k^{+}, p\right)$ (see Corollary 10.3 .11 of [22]). Note that for each $n \geq 0$, the intermediate field $k_{n}^{+}$of $k_{\infty}^{+ \text {cyc }}=k^{+} \mathbb{Q}_{\infty}^{\text {cyc }}$ is totally real. Hence from Theorem 1.2 and the first claim of the corollary, we can prove the second claim of the corollary. This completes the proof of the corollary.

When the number $r_{k}(p)$ of primes of $k$ dividing $p$ is one, we have the following lemma which is well known. We prove this using a remark of Iwasawa.

Lemma 3.13. For a number field $k$, if $r_{k}(p)=1$, then the generalized Gross conjecture holds for $(k, p)$.

Proof. Let $u\left(k_{\infty} / k\right)$ be the set of primes of $k$ which are ramified in $k_{\infty} / k$. Then it follows from the elementary Iwasawa theory and the class field theory that $u\left(k_{\infty} / k\right)$ is a nonempty subset of the set of primes of $k$ dividing $p$. By the assumption of the lemma, the cardinality $\sharp\left(u\left(k_{\infty} / k\right)\right)$ is equal to one for any $\mathbb{Z}_{p}$-extension $k_{\infty}$ of $k$. We recall the observation of Iwasawa that a $\mathbb{Z}_{p}$-extension $k_{\infty}$ of $k$ such that $\sharp\left(u\left(k_{\infty} / k\right)\right)$ is minimum in the family of all $\mathbb{Z}_{p}$-extensions of $k$ must satisfy one of the equivalent conditions (i),(ii) and (iii) of $\S 2$ (see p. 804 of [13]). By taking $k_{\infty}=k_{\infty}^{\text {cyc }}$, we see that the generalized Gross conjecture holds for $(k, p)$. This completes the proof of the lemma. 
Remarks. 1. For every $\mathbb{Z}_{p}$-extension of $k$, there exists an integer $n(k) \geq 0$ such that every prime which ramifies in $k_{\infty} / k_{n(k)}$ is totally ramified (see Lemma 13.3 of [25]). Hence note that for all $n \geq n(k)$, the number $r_{\infty}(p)$ of primes of $k_{\infty}$ lying over $p$ is equal to that of $k_{n}$, i.e., $r_{\infty}(p)=r_{k_{n}}(p)$.

2. As special cases of Corollary 3.12, if the real subfields in (i) and (ii) of Corollary 3.12 have only one prime lying over $p$, then Lemma 3.13 provides us with more simple descriptions.

(i) If $k$ is a totally real field with $r_{\infty}(p)=1$, then the affirmation of the Leopoldt conjecture for $\left(k_{\infty}^{\mathrm{cyc}}, p\right)$ is equivalent to Hilbert's theorem 90 for $\overline{\Delta\left(U_{n}(p)\right)}$ over $k_{\infty}^{\text {cyc }} / k_{n}$ for all $n \geq 0$.

(ii) If $k$ is a CM-field with the maximal real subfield $k^{+}$such that $r_{\infty^{+}}(p)=$ 1 , then the affirmation of the Leopoldt conjecture for $\left(k_{\infty}^{\text {cyc }}, p\right)$ is equivalent to Hilbert's theorem 90 for $\overline{\Delta\left(U_{k_{n}^{+}}(p)\right)}$ over $k_{\infty}^{+ \text {cyc }} / k_{n}^{+}$for all $n \geq 0$.

3 . Now let $k$ be an arbitrary number field which is not necessarily totally real with $r_{\infty}(p)=1$. We notice that due to Lemma 3.13, if $r_{\infty}(p)=1$, then the affirmation of the Leopoldt conjecture for $\left(k_{\infty}^{\mathrm{cyc}}, p\right)$ is still equivalent to Hilbert's theorem 90 for $\overline{\Delta\left(U_{n}(p)\right)}$ over $k_{\infty}^{\text {cyc }} / k_{n}$ for all $n \geq 0$. This statement can also be recovered in the proof of Theorem 1.2. In fact the proof of Theorem 1.2 use the generalized Gross conjecture essentially only for (3) in the proof of Theorem 1.2 which is automatically satisfied when $r_{\infty}(p)=1$.

4. Finally let $k$ be an arbitrary number field which is not necessarily totally real with $r_{\infty}(p)>1$. In this case, we may apply a result of [24] with the assumption that $k$ is Galois containing $\mu_{p}$ and $r_{\infty}(p)=2$. In this case the result shows that the generalized Gross conjecture holds for $\left(k_{\infty}^{\mathrm{cyc}}, p\right)$. In fact if $k$ is Galois containing $\mu_{p}$ with $r_{\infty}(p)=2$, then it follows that for each $n \geq 0, k_{n}$ is also Galois containing $\mu_{p}$ and $r_{k_{n}}(p)=2$. Hence if $k$ is Galois containing $\mu_{p}$ with $r_{\infty}(p)=2$, then the affirmation of the Leopoldt conjecture for $\left(k_{\infty}^{\mathrm{cyc}}, p\right)$ is equivalent to Hilbert's theorem 90 for $\overline{\Delta\left(U_{n}(p)\right)}$ over $k_{\infty}^{\text {cyc }} / k_{n}$ for all $n \geq 0$.

\section{6.}

We are informed that results of Jaulent (cf. [15] and [16]) can also be used to recover a part of Theorem 1.2 using results of [18] and [24]. Under the assumption of the generalized Gross conjecture for $\left(K_{\infty}^{\text {cyc }}, p\right)$, we will show that the Leopoldt conjecture for $\left(K_{\infty}^{\mathrm{cyc}}, p\right)$ implies the condition of Hilbert's theorem 90 for $\overline{\Delta\left(U_{k}(p)\right)}$ over $k_{\infty}^{\text {cyc }} / k$. 
We use the logarithmic description introduced by Jaulent in [15] to compute here the $\mathbb{Z}_{p}$-rank of $\Omega$. Let $r_{1}, r_{2}$ and $s$ be the numbers of real places, complex places and respectively places dividing $p$ in $K$. We use the following definitions from [15].

(i) $\mu_{K}$ the $p$-subgroup of the group of roots of unity in $K$;

(ii) $\mathcal{E}_{K}^{\prime}=\mathbb{Z}_{p} \otimes_{\mathbb{Z}} E_{K}^{\prime}$ the $p$-adification of the group of $p$-units $E_{K}^{\prime} \cong$ $\mu_{K} \mathbb{Z}^{r_{1}+r_{2}+s-1}$

(iii) $\widetilde{\mathcal{E}}_{K}$ the subgroup of logarithmic units of $K$;

(iv) $\mu_{K}^{l o c}=\left(\mathbb{Z}_{p} \otimes K^{\times}\right) \cap \prod \mu_{\mathfrak{p}}$;

(v) $\mathcal{R}_{p}=\prod_{\mathfrak{p} \mid p} \mathcal{R}_{\mathfrak{p}}$ the $p$-adic compactification of the semi-local product $\prod_{\mathfrak{p} \mid p} K_{\mathfrak{p}}$

(vi) $\mu_{p}=\prod_{\mathfrak{p} \mid p} \mu_{\mathfrak{p}}$ the torsion subgroup of $\mathcal{R}_{p}$.

From the proposition 1.1 of [24], it follows that the $\Gamma$-invariant $T_{p}(K)^{\Gamma}$ of the Tate module $T_{p}(K)$ of $K$ is given by

$$
T_{p}(K)^{\Gamma}=\frac{\widetilde{\mathcal{E}}_{K}}{\left(U_{K}(p) \otimes \mathbb{Z}_{p}\right)^{\mathrm{univ}}} .
$$

If we denote by $\delta_{G}(K)$ the defect of the generalized Gross conjecture(GGC) of $K$, i.e.,

$$
\delta_{G}(K):=\operatorname{rank}_{\mathbb{Z}_{p}} T_{p}(K)
$$

then it follows from Theorem 3.7 that

$$
\widetilde{\mathcal{E}}_{K} \cong \mu_{K} \mathbb{Z}_{p}^{r_{1}+r_{2}+\delta_{G}(K)}
$$

This is in fact Proposition 3.4 of [15].

Since $\mu_{K}^{l o c}$ is the kernel of the semi-localization homomorphism

$$
s_{p}: \widetilde{\mathcal{E}}_{K} \longrightarrow \mathcal{R}_{p} / \mu_{p}
$$

we can write

$$
\mu_{K}^{l o c} \cong \mu_{K} \mathbb{Z}_{p}^{\delta_{L}(K)}
$$

where $\delta_{L}(K)$ denotes the defect of the Leopoldt conjecture(GLC) of $K$ (cf. Scolie 2.13 of [16]) and hence

$$
s_{p}\left(\widetilde{\mathcal{E}}_{K}\right) \cong \widetilde{\mathcal{E}}_{K} / \mu_{K}^{l o c} \cong \mathbb{Z}_{p}^{r_{1}+r_{2}-\left(\delta_{L}(K)-\delta_{G}(K)\right)}
$$


where $s_{p}\left(\widetilde{\mathcal{E}}_{K}\right)$ is just the group $\Omega$. Hence we obtain immediately the following statement. If one assumes $r k_{\mathbb{Z}_{p}}(\Omega)=r k_{\mathbb{Z}_{p}}\left(s_{p}\left(\widetilde{\mathcal{E}}_{K}\right)\right)=r_{1}+r_{2}$, then it follows that

$$
\delta_{L}(K)=\delta_{G}(K) .
$$

In special this implies that (GLC) $\Longleftrightarrow$ (GGC).

Moreover under the assumption $(\mathrm{GGC})$ for $\left(K_{\infty}^{\mathrm{cyc}}, p\right)$, we will show that (GLC) for $\left(K_{\infty}^{\text {cyc }}, p\right)$ implies the condition of Hilbert's theorem 90(H90) for $\overline{\Delta\left(U_{k}(p)\right)}$ over $k_{\infty}^{\text {cyc }} / k$. Put $G_{n}:=G\left(K_{n} / K\right)$. By Proposition 2 of [9], the generalized Gross conjecture for $\left(K_{\infty}^{\mathrm{cyc}}, p\right)$ implies that the quotients $\widetilde{\mathcal{E}}_{K_{n}} / \mu_{K_{n}}$ are free over the group algebra $\mathbb{Z}_{p}\left[G_{n}\right]$ of rank $r_{1}+r_{2}$. Thus, in this case, the logarithmic groups $\widetilde{\mathcal{E}}_{K_{n}}$ have trivial cohomology.

It follows from the weak Leopoldt conjecture over $K_{\infty}^{\text {cyc }} / K$ that the defect $\mu_{K_{n}}^{\text {loc }} / \mu_{K_{n}}$ of the Leopoldt conjecture stabilizes in $K_{\infty}^{\text {cyc }} / K$.

Hence the exact sequence

$$
1 \longrightarrow \mu_{K_{n}}^{l o c} \longrightarrow \widetilde{\mathcal{E}}_{K_{n}} \longrightarrow s_{p}\left(\widetilde{\mathcal{E}}_{K_{n}}\right) \longrightarrow 1
$$

and the well known fact that $H^{i}\left(G_{n}, \mu_{K_{n}}\right)=1$ for all $i \geq 0$ result in the following isomorphisms

$$
H^{1}\left(G_{n}, s_{p}\left(\widetilde{\mathcal{E}}_{K_{n}}\right)\right) \cong H^{2}\left(G_{n}, \mu_{K_{n}}^{l o c}\right)=H^{2}\left(G_{n}, \mu_{K_{n}}^{l o c} / \mu_{K_{n}}\right) \cong\left(\mathbb{Z}_{p} / p^{n} \mathbb{Z}_{p}\right)^{\delta_{L}\left(K_{\infty}^{\text {cyc }}\right)} .
$$

It follows that

$$
H^{1}\left(G_{n}, s_{p}\left(\widetilde{\mathcal{E}}_{K_{n}}\right)\right)=1 \Longleftrightarrow \delta_{L}\left(K_{n}\right)=0 .
$$

By taking inverse limits to the exact sequence of compact modules obtained from $H^{1}\left(G_{n}, s_{p}\left(\widetilde{\mathcal{E}}_{K_{n}}\right)\right)=1$,

$$
1 \longrightarrow\left(\gamma_{n}-1\right) s_{p}\left(\widetilde{\mathcal{E}}_{K_{n}}\right) \longrightarrow s_{p}\left(\widetilde{\mathcal{E}}_{K_{n}}\right) \longrightarrow N_{n} s_{p}\left(\widetilde{\mathcal{E}}_{K_{n}}\right) \longrightarrow 1
$$

it follows that

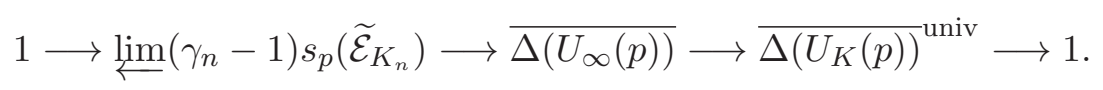

The exact sequence $1 \longrightarrow \operatorname{ker}(\gamma-1) \longrightarrow s_{p}\left(\widetilde{\mathcal{E}}_{K_{n}}\right) \stackrel{\gamma-1}{\longrightarrow}(\gamma-1) s_{p}\left(\widetilde{\mathcal{E}}_{K_{n}}\right) \longrightarrow 1$ leads to

$$
(\gamma-1) \overline{\Delta\left(U_{\infty}(p)\right)} \cong \varliminf_{\longleftarrow}\left((\gamma-1) s_{p}\left(\widetilde{\mathcal{E}}_{K_{n}}\right)\right.
$$

since $\underset{\lim }{\longleftarrow} \operatorname{ker}(\gamma-1)=1$. Hence we have

$\delta_{L}\left(K_{n}\right)=0$ for all $n \geq 0 \Longleftrightarrow H^{1}\left(G_{n}, s_{p}\left(\widetilde{\mathcal{E}}_{K_{n}}\right)\right)=1$ for all $n \geq 0 \Longrightarrow(H 90)$. 
For the reverse direction $(H 90) \Longrightarrow \delta_{L}\left(K_{n}\right)=0$, we need to apply again $\S 3.3$.

\section{Acknowledgments}

We would like to thank the referee for helpful comments and suggestions.

\section{References}

[1] E. Artin and J. Tate, Class field theory (2nd edition). Addison-Wesley, 1990.

[2] F. Bertrandias and J. Payan, $\Gamma$-extensions et invariants cyclotomiques. Ann. Sci. Ecole Norm. Sup., 5 (1972), 517-543.

[3] N. Bourbaki, Elements of Mathematics: General Topology Part 1. Addison-Wesley, 1966.

[4] N. Bourbaki, Elements of Mathematics: Commutative Algebra. Addison-Wesley, 1972.

[5] A. Brumer, Galois groups of extensions of algebraic number fields with given ramification. Michigan Math. J., 13 (1966), 33-40.

[6] L. Federer and B. Gross (with an appendix by W. Sinnott), Regulators and Iwasawa modules. Invent. Math., 62 (1981), 443-457.

[7] G. Gras, Class field theory. Springer-Verlag, 2003.

[8] R. Greenberg, On a certain l-adic representation. Invent. Math., 21 (1973), 117-124.

[9] C. Greither, Sur les normes universelles dans les $\mathbb{Z}_{p}$-extensions. J. Théor. Nombres Bordeaux (1994), 205-220.

[10] B. Gross, p-adic L-series at $s=0$. J. Fac. Sci. Univ. Tokyo IA, 28 (1981), 979-994.

[11] T. Husain, Introduction to Topological Groups. W. B. Saunders Company (1966).

[12] K. Iwasawa, On $Z_{l}$-extensions of algebraic number fields. Ann. of Math., 98 (1973), 246-326.

[13] K. Iwasawa, On cohomology groups of units for $Z_{p}$-extensions. Amer. J. Math., 105 (1983), 189-200. 
[14] J.-F. Jaulent, L'arithmetique des l-extensions. Thèse, Université de Franche Comté, 1986.

[15] J.-F. Jaulent, Classes logarithmiques des corps de nombres. J. Théorie des Nombres de Bordeaux, 6 (1994), 301-325.

[16] J.-F. Jaulent, Théorie l-adique globale du corps de classes. J. Théorie des Nombres de Bordeaux, 10 (1998), 355-397.

[17] M. Kolster, An idelic approach to the wild kernel. Invent. Math., 103 (1991), 9-24.

[18] L. Kuz'min, The Tate module of algebraic number fields. Izv. Akad. Nauk SSSR Ser. Mat., 36 (1972), 267-327.

[19] S. Lang, Cyclotomic Fields I and II (with an appendix by K. Rubin). GTM, Vol. 121, Springer-Verlag, 1990.

[20] H. Leopoldt, Zur Arithmetik in abelschen Zahlkörpern. J. Reine Angew. Math., 209 (1962), 54-71.

[21] J. Neukirch, Class field theory. Grundlehren der Math., Vol. 280, Springer-Verlag, 1986.

[22] J. Neukirch, A. Schmidt and K. Wingberg, Cohomology of Number Fields (2nd edition). Springer-Verlag, 2008.

[23] L. Pontriagin, Topological Groups (2nd edition). Gordon and Breach, 1966.

[24] S. Seo, On the Tate module of a number field. Preprint.

[25] L. Washington, Introduction to Cyclotomic Fields (2nd edition). GTM, Vol. 83, Springer-Verlag, 1997.

Department of Mathematics, Yonsei University

50 Yonsei-ro, Seodaemun-Gu, Seoul 120-749, Korea

E-mail address: sgseo@yonsei.ac.kr

RECEIVED JANUARY 1, 2014 